\title{
Content Analysis of Library \& Information Science (LIS) Curricula in Pakistani Universities
}

\author{
Farhat Hussain* \\ Munira Nasreen Ansari**
}

\begin{abstract}
Education in Library \& Information Science at postgraduate level in Pakistan is imparted by 12 universities including nine in public and three in private sector, whereas one public university has suspended the program in 2008. This paper aims to analyze the courses offered by the library schools of Pakistan. The study explores the number and type of courses being taught in a year/semester. The number of courses offered in each semester is more or less equal in all the universities except Peshawar University which is practicing annual system. All the universities offer core and technical courses which are the requirement of a professional degree. The specialized and elective courses are fairly different but majority of the courses are same. Most of the elective courses meet the local needs of the library schools. To fulfill the national and international demands, almost all the library schools are designing and offering more and more ICTrelated courses. They are also paying special attention towards practical component of the courses while designing and developing curriculum. All library schools are trying their best to adopt modern teaching methods and facilities. All possible assessment techniques are applied to evaluate the students' performance. Hence it can be said that the library education and the courses offered by the library schools in Pakistan are of superb quality and meeting the challenges of international market.
\end{abstract}

Keywords: Library Education; Library \& Information Science; LIS Education in Pakistan; Library Schools; LIS courses

* Assistant Professor,Department of Library and Information Science, University of Karachi

** Associate Professor,Department of Library and Information Science,University of Karachi 


\section{Introduction}

The history of library education in united India (including today's Pakistan) begins from Baroda state in 1911. Through the struggles of Maharaja Sayajrao III, Gaekwar of Baroda (1862-1939) library schools were introduced. He was a civilized and educated ruler who supported and promoted libraries. According to Nagar", "He called and welcomed William Alanson Borden, a leading American librarian, who had been a pupil-assistant of Charles Ammi Cutter at the Boston Athenaeum and a lecturer-associate of Melvil Dewey at Columbia University's School of Library Economy."

Asa Don Dickinson was another American academic library pioneer in British India who was invited by the University of the Punjab in Lahore for a year during 1915-16 "to organize its library on modern American lines." [Dr] Khurshid ${ }^{2}$ wrote "Some of the developments emanating from British rule significantly differed from the practice then existing in Great Britain itself. For example, the Commission of Asa Don Dickinson in 1915 specifically required him to organize the library-training class of Punjab University at Lahore. Such training did not exist at all in any British universities at that time. The Calcutta University Commission Report (1917-19) on the other hand, stressed the need for appointing a trained librarian with the status and rank of a professor at Calcutta University. Even this practice was uncommon in British universities where preference for such an appointment was given to those with academic qualifications."

The significant change of American influence at the Punjab University during 1913 occurred due to the appointment of Professor James C.R. Ewing as the vice chancellor of the University of the Punjab (1910-17). He was an American citizen and had been educated in the United States. As the vice chancellor, he submitted some proposals to the syndicate of the university to allocate "a sum of Rs. 65000 provided by the Government of India for the improvement of the functioning of the University." (Anwar3)

Recruitment of a professional librarian to completely organize the library and to teach young class for library procedures was suggested by Ewing. His suggestion was implemented in selecting Dickinson for the position of a temporary university librarian. Dickinson had “received a year's training under Melvil Dewey in the New York State Library. Subsequently, he had 10 years varied experience in library work including 3 years in the Brooklyn Public Library and 3 years in the Washington State

1 Nagar, Murari Lal. Foundation of Library Movement in India. Ludhiana: Indian Library Institute and Bibliographical Center, 1983. p. 2-3.

2 Khurshid, Anis. Library Education in South Asia. 20 (1-2). 1970. pp. 59-60.

3 Anwar, Mumtaz Ali. Asa Don Dickinson: The Founding Father of Modern Librarianship in British India. Pakistan Library Bulletin. 21 (2), 1990. p. 13. 
College Library." (Anwar ${ }^{4}$ ). At that time training in librarianship was not available in the British universities. Describing the status of the university librarian in 1915, Anwar ${ }^{5}$ wrote, "Dickinson was the first highly educated and professionally trained individual to be appointed as the university librarian in any of the universities in British India. One could extend this exceptional development to most of the British Empire including Great Britain and the British colonies in Asia and Africa." According to Khurshid 6 , the curriculum introduced by Dickinson covered basic subjects including decimal classification, cataloguing rules, list of subject headings, dictionary catalogue, and open shelves. The syllabus was enlarged in 1921 by Labhu Ram, a student of Dickinson and the assistant librarian of the University of the Punjab, to include seventy-two lectures instead of the original twenty-five. In 1921, the subjects used to be taught included: 1) foreign languages 2) linguistic survey of India 3) basic selection 4) law of copyright in England and India 5) a survey from Tennyson to Bernard Shaw 6) sources of provincial histories of India 7) oriental bibliography 8) milestones of English literatures 9) library buildings, their designs and equipment 10) Anglo-Indian literatures, and 11) open access and technical libraries.

The education of library and information science at postgraduate level is being offered by the following nine public sector universities of Pakistan:

1. University of Karachi, Karachi

2. University of the Punjab, Lahore

3. University of Peshawar, Peshawar

4. University of Sindh, Jamshoro

5. University of Baluchistan, Quetta

6. Islamia University, Bahawalpur

7. Allama Iqbal Open University, Islamabad

8. University of Sargodha, Sargodha

9. Khushal Khan Khattak University, Karak
(2015)

* Short Certificate course started in 1915, Postgraduate program was started in 1959.

** At the time of establishment, the University was an examining and degree awarding body,

but later on, in 1951, it got the status of an academic and research institution. The Department

was established in 1965 with certificate course, but postgraduate program in Library Science

was started in 1970.

ibid

ibid

Khurshid, Anis. Library Education in South Asia. 20 (1-2). 1970. p. 60. 
*** The Library Science program was started in 1985 with an undergraduate short certificate

course in Library Science but a regular on-campus Department was established in 2001.

Besides the above nine public sector universities of Pakistan, Bahauddin Zakariya University, Multan had also started postgraduate program in Library \& Information Science in 2004, but after four years i.e. in 2008 the program was shelved due to some administrative reasons. Till now this program is suspended. This university had adopted the same scheme of studies being practiced by the University of Punjab.

Three universities in private sector; Minhaj University, Lahore, Superior University, Lahore and Sarhad University of Science and Technology, Peshawar have also been offering the MLIS/M.Phil program since 2014 but as the scope of this paper is confined to only public sector universities of Pakistan so they have not been included here.

\section{Objectives}

The objectives of the study are:

1. To find out the programs, teaching methods, teaching facilities/aids, and system of examinations of all Pakistani public sector universities offering education in LIS.

2. To examine the LIS postgraduate courses taught at Pakistani public sector library schools.

3. To review the course contents so that the need of the future demands in present technological revolution era can be determined.

4. To find out the ratio between the theory and practical courses.

5. To find out the status of core and elective courses.

6. To collect information about the modern especially ICT-related courses offered in the curricula.

\section{Significance and Implications}

Although a number of research articles have been contributed on the curricula being offered at Pakistani library schools but the course contents have not been deeply studied and analyzed. This article will attempt to analyze the contents of the courses from different angles to determine the ongoing demands of modern and technological-related courses in the field of librarianship. The article will provide the library schools a foundation to design their courses accordingly. 


\section{Literature Review}

Browsing of the literature reveals that much has been written on every aspect of LIS curricula being taught at Pakistani library schools. Suggestions have also been given to improve the abilities and proficiencies of the library professionals and to incorporate these recommendations in the curricula for better production of LIS graduates.

Khan and Mahmood ${ }^{7}$ compare Pakistani LIS Schools curricula with HEC-designed curriculum for LIS schools in Pakistan. The authors reveal that only one LIS school follows HEC-designed curriculum while rest of the schools have set their own standards. The study has defined the weak areas (that all the courses recommended by HEC are not being offered by all the library schools) that suggests more refinements in order to qualify the competition nationally among LIS schools in Pakistan.

Mahmood $^{8}$ studies LIS curriculum for seeking the feedback of the senior librarians and employers of young librarians. The findings reveal that "Competencies required of an LIS graduate can be divided into three categories: LIS core, management, and ICTs. All three areas should be given a balanced importance in the curriculum". As a result of these surveys, the author assessed educational needs of entry level and experienced manpower and also gave recommendations.

Warraich ${ }^{9}$ analyzed the curriculum of MLIS at University of Punjab and conducted a survey on the alumni of the department to find out their satisfaction about the curricula they studied. Some of the problems or deficiencies pointed out in the survey include: Non-proficiency in written and spoken English, lack of knowledge of office communication, lack of interviewing skills, unawareness of advanced library softwares, lack of IT skills, poor interpersonal skills, lack of leadership skills, weak presentation skills, lack of research skills etc. The respondents suggested that if these shortcomings are removed and are incorporated in the curriculum, the alumni will get better job opportunities and will compete the market.

7 Khan, M. Tufail and Mahmood, Khalid. A comparison of MLIS curricula taught in Pakistani library and information science schools with Higher Education Commission curriculum. Library Philosophy and Practice, 2013. Retrieved from: http://works.bepress.com/khalid mahmood/18/ on 12 August 2017.

8 Mahmood, Khalid. LIS Curriculum Review Using Focus Group Interviews of Employers. Library Philosophy and Practice, 2012. Retrieved from http://digitalcommons.unl.edu/libphilprac /756/ on 12 August 2017.

9 Warraich, Nosheen Fatima. MLIS Curriculum at Punjab University: Perception and Reflections. Library Philosophy and Practice, (e-Journal), March 2010. 
Discussing the merits and demerits of the courses of Library Management taught by all the library schools of Pakistan, Ameen ${ }^{10}$ observes that library leadership is almost an unfamiliar area in library and information science (LIS) education. The author spots the barriers faced in the evolution of leadership traits among professionals. Findings suggest that information professionals today require quality of leadership and anticipation to innovate the future through planning and preventing from unexpected changes in such emerging social, technological and professional scenario of the country.

Haider 11 analyzes six LIS Departments of the country with respect to their cataloging and classification courses' contents and teaching methodologies. The study reveals that in almost all library schools of the country, there is a severe scarcity of DDC and LCC schedules of classification schemes. Although latest edition of DDC is purchased but only 8-10 sets are acquired due to shortage of financial resources. Students do practice in groups and 2-3 students share one set of the schedule. Suggestions comprise: curricula revision, organizing training of teachers for cataloging and classification courses in developed countries, improvement of laboratories, and the organization of continuing education programs.

Yusuf 12 analyzes the courses offered in BLIS and MLIS in six library schools in Pakistan (Universities of Karachi, Baluchistan, Bahawalpur, Peshawar, Punjab and Sindh). He suggests that all library schools should offer one standard curricula and necessary process should be initiated by University Grants Commission (UGC). After reviewing briefly the programs and courses of all six library schools of that time, succinct comments were given on the shortcomings in the curricula. Recommendations were made to include courses on communication techniques, statistics, research methods, networking databases, selective dissemination of information in the curricula of the universities.

Sabzwari13 discusses the situation of library education in Pakistan in detail. He reviews the postgraduate courses in library schools in four universities of Pakistan which are Karachi, Punjab, Peshawar and Sindh Universities. With respect to curricula, he advises that at postgraduate diploma level (BLS, DLS, or M.A. Previous),

10 Ameen, Kanwal. Challenges of preparing LIS professionals for leadership roles in Pakistan. Journal of Education for Library and Information Science. 47(3), 2006. pp.200217.

11 Haider, S. J. Teaching of Cataloging and Classification in Pakistan. Cataloging \& Classification Quarterly, 43, 2006. pp. 53-65.

12 Yusuf, M. (1996) Critical view of the courses offered at library schools in Pakistan. PLA journal. 18, 1996. pp. 68-76.

13 Sabzwari, G.A. Library education and manpower needs in Pakistan. Pakistan library bulletin. IV (3-4), 1972. pp. 55-71. 
specialization in the profession should be offered and there should be an optional course of school, college or children librarianship and students should opt any one of them as per their interest or ability. At Masters level, he suggests to include the courses which should fulfill the local market needs. For this purpose, he proposes the courses on Industrial, Medical and Commercial Librarianship in Karachi whereas the courses of Scientific, Agricultural and Governmental Librarianship in Lahore, Peshawar and Hyderabad. He recommends that library schools should make the arrangement of In-Service Training on regular basis. He further suggests that library schools should provide the facility of limited time registration as external candidate for those senior library professionals who require improvement in their skills and qualification for better services.

After going through the articles and literature on LIS curricula in Pakistan, it is concluded that all the library schools of the country are offering courses of their own accord and needs and rarely there are one or two schools that are following the curriculum designed by HEC. The articles also give some suggestions to maintain the international standard and to fulfill the market needs. The most important shortcomings which are pointed out in the curricula include non-adoption of technological and environmental development as the job-offering organizations find lack of knowledge and skills about the modern innovations especially ICT in the prospective candidates. Another deficiency is verbal and written English communication. No doubt, it is not a professional weakness but it is expected a necessary ability for a librarian. Other deficiencies are unawareness about the latest library softwares and lack of leadership skills. To remove these shortcomings and enhance professional skills, the curriculum should be revised and updated by incorporating the relevant topics so that better job opportunities be acquired.

\section{Methodology}

The curricula of all the library schools of Pakistan were collected either directly from the chairperson of the department or from the websites of the universities or departments. These courses and their contents were thoroughly studied with different angles like number of courses offered in a year/semester, titles of courses, traditional and modern courses, ICT-related trends in the curricula, elective courses, practical component in the curricula, teaching methods, students' evaluation/assessment methods, teaching facilities/aids etc. This exercise developed a clear picture of the strengths and weaknesses of all the departments with respect to the curricula and teaching methods and facilities.

The documents and online resources relating to the introduction, history, programs, courses offered by each library school of Pakistan were also searched. For this purpose, the information available on the websites of the concerned universities and the library schools were downloaded and personal contacts were also made 
through telephone, e-mail, and surface mail. The faculty members and especially the chairpersons of all the departments provided the same material.

\section{Findings and Discussion}

\section{Programs Offered}

The universities of Pakistan are offering four different graduate and postgraduate programs in Library and Information Science. The following table elaborates the same programs:

Table-1 LIS Programs offered by Universities of Pakistan

\begin{tabular}{|c|c|c|c|c|c|}
\hline S.No. & Name of University & $\begin{array}{c}\text { BS } \\
4 \text { years }\end{array}$ & $\begin{array}{c}\text { BLIS } \\
1 \text { year }\end{array}$ & $\begin{array}{l}\text { MLIS } \\
1 \text { year }\end{array}$ & $\begin{array}{c}\text { MLIS } \\
2 \text { years }\end{array}$ \\
\hline 1. & University of Karachi & $\mathrm{x}$ & $\checkmark$ & $\checkmark$ & $\mathrm{x}$ \\
\hline 2. & $\begin{array}{l}\text { University of the Punjab, } \\
\text { Lahore }\end{array}$ & $\mathrm{x}$ & $\mathrm{x}$ & $\mathrm{x}$ & $\checkmark$ \\
\hline 3. & $\begin{array}{l}\text { University of Sindh, } \\
\text { Jamshoro }\end{array}$ & $\checkmark$ & $\mathrm{x}$ & $\mathrm{x}$ & $\mathrm{x}$ \\
\hline 4. & University of Peshawar & $\mathrm{X}$ & $\mathrm{x}$ & $\mathrm{x}$ & $\checkmark$ \\
\hline 5. & $\begin{array}{l}\text { University of Baluchistan, } \\
\text { Quetta }\end{array}$ & $\mathrm{X}$ & $\mathrm{x}$ & $\mathrm{x}$ & $\checkmark$ \\
\hline 6. & $\begin{array}{ll}\text { Islamia } & \text { University } \\
\text { Bahawalpur } & \\
\end{array}$ & $\checkmark$ & $\mathrm{x}$ & $\mathrm{x}$ & $\checkmark$ \\
\hline 7. & $\begin{array}{ll}\text { Allama Iqbal } & \text { Open } \\
\text { University, Isl. } & \\
\end{array}$ & $\mathrm{X}$ & $\mathrm{x}$ & $\mathrm{x}$ & $\checkmark$ \\
\hline 8. & Sargodha University & $\checkmark$ & $\mathrm{x}$ & $\mathrm{x}$ & $\checkmark$ \\
\hline 9. & $\begin{array}{l}\text { Khushal Khan Khattak } \\
\text { University }\end{array}$ & $\checkmark$ & $\mathrm{x}$ & $\mathrm{x}$ & $\checkmark$ \\
\hline
\end{tabular}

All the universities of Pakistan are offering different graduate and postgraduate programs in Library and Information Science. University of Karachi offers one-year postgraduate BLIS program which is equivalent to M.A. (previous) or postgraduate diploma in the same university or other universities. After successful completion of one-year BLIS, the students are eligible to continue one-year MLIS program. The University of the Punjab, Lahore offers two-year Masters program in Information Management which is equivalent to MLIS or MA-LIS programs of other universities. University of Sindh, Jamshoro (Hyderabad) offers 4-year BS program to students having HSC or Intermediate. University of Peshawar offers 2-year Masters program whereas University of Baluchistan, Quetta and Allama Iqbal Open University, Islamabad are also offering 2-year Masters program. Islamia University, Bahawalpur, Sargodha University and Khushal Khan Khattak University, Karak are offering 4-year BS and 2-year Master programs simultaneously. 


\section{System of Examinations}

Both semester and annual systems are in practice in the universities of Pakistan.

Table-2 Examination Systems adopted by the universities

\begin{tabular}{|c|l|c|c|c|c|}
\hline S.No. & \multicolumn{1}{|c|}{ Name of University } & Semester & Annual & \multicolumn{2}{|c|}{ Total Semesters } \\
\cline { 3 - 5 } & & & & MLIS & BS \\
\hline 1. & University of Karachi & $\checkmark$ & $\mathrm{x}$ & $2+2$ & $\mathrm{x}$ \\
\hline 2. & $\begin{array}{l}\text { University of the Punjab, } \\
\text { Lahore }\end{array}$ & $\checkmark$ & $\mathrm{x}$ & 4 & $\mathrm{x}$ \\
\hline 3. & $\begin{array}{l}\text { University of Sindh, } \\
\text { Jamshoro }\end{array}$ & $\checkmark$ & $\mathrm{x}$ & $\mathrm{x}$ & 8 \\
\hline 4. & $\begin{array}{l}\text { University of Peshawar } \\
\text { University of Baluchistan, } \\
\text { Quetta }\end{array}$ & $\checkmark$ & $\mathrm{x}$ & 4 & $\mathrm{x}$ \\
\hline 6. & $\begin{array}{l}\text { Islamia University } \\
\text { Bahawalpur }\end{array}$ & $\checkmark$ & $\mathrm{x}$ & 4 & 8 \\
\hline 7. & $\begin{array}{l}\text { Allama Iqbal Open } \\
\text { University, Isl. }\end{array}$ & $\checkmark$ & $\mathrm{x}$ & 4 & $\mathrm{x}$ \\
\hline 8. & $\begin{array}{l}\text { Sargodha University } \\
\text { Khushal Khan Khattak } \\
\text { University }\end{array}$ & $\checkmark$ & $\mathrm{x}$ & 4 & 8 \\
\hline
\end{tabular}

Of nine universities offering Library and Information Science, eight universities are pursuing semester system whereas remaining only one i.e. Peshawar University is practicing annual system. Of them, six universities are offering MLIS or Masters in Library \& Information Science or MA in Information Management, one university (Karachi University) offers BLIS and MLIS programs of one year each, and one university (Sindh University) imparts four year BS program. Islamia University Bahawalpur, Sargodha University, and Khushal Khan Khattak University, Karak are offering both two and four year programs concurrently.

\section{Curriculum offered by Pakistani LIS Schools}

The integral part of any educational program is curriculum. The core curriculum should have the ability to meet the needs of LIS profession. This is composed of the following:

1. The objectives of program

2. Number of courses

3. Outline of the courses 
Jhss, Vol. 9, No. 1 , January to June, 2018

4. Practical contents

The curriculum should carry the contents of each course and it should also explain the learning outcomes of the course that after successful completion of the program what would be expected from the learner.

Various number of courses are taught in every university of Pakistan to complete the educational program in library and information science/information management.

Table-3 Number of courses offered by the universities

\begin{tabular}{|c|c|c|c|c|c|c|c|}
\hline \multirow[t]{2}{*}{ S.No. } & \multirow{2}{*}{$\begin{array}{l}\text { NAME OF } \\
\text { UNIVERSITY }\end{array}$} & \multicolumn{4}{|c|}{ SEMESTER SYSTEM } & \multicolumn{2}{|c|}{$\begin{array}{l}\text { ANNUAL } \\
\text { SYSTEM }\end{array}$} \\
\hline & & $\begin{array}{l}1^{\text {st }} \\
\text { Semester }\end{array}$ & $\begin{array}{l}2^{\text {nd }} \\
\text { Semester }\end{array}$ & $\begin{array}{l}3^{\text {rd }} \\
\text { Semester }\end{array}$ & $\begin{array}{l}4^{\text {th }} \\
\text { Semester }\end{array}$ & $\begin{array}{l}1^{\text {st }} \\
\text { Year }\end{array}$ & $\begin{array}{l}2^{\text {nd }} \\
\text { Year }\end{array}$ \\
\hline 1. & $\begin{array}{l}\text { University of } \\
\text { Karachi }\end{array}$ & 6 & 7 & 7 & 7 & $\mathrm{x}$ & $\mathrm{x}$ \\
\hline 2. & $\begin{array}{l}\text { University of } \\
\text { the Punjab }\end{array}$ & 7 & 6 & 6 & 6 & $\mathrm{x}$ & $\mathrm{x}$ \\
\hline 3. & $\begin{array}{l}\text { University of } \\
\text { Sindh* }\end{array}$ & $\mathrm{x}$ & $\mathrm{x}$ & $\mathrm{x}$ & $\mathrm{x}$ & $\mathrm{x}$ & $\mathrm{x}$ \\
\hline 4. & $\begin{array}{l}\text { University of } \\
\text { Peshawar }\end{array}$ & $\mathrm{x}$ & $\mathrm{x}$ & $\mathrm{x}$ & $\mathrm{x}$ & 6 & 6 \\
\hline 5. & $\begin{array}{l}\text { University of } \\
\text { Baluchistan }\end{array}$ & 5 & 5 & 6 & 4 & $\mathrm{x}$ & $\mathrm{x}$ \\
\hline 6. & $\begin{array}{l}\text { Islamia } \\
\text { University } \\
\text { Bahawalpur* }\end{array}$ & 6 & 6 & 6 & 6 & $\mathrm{x}$ & $\mathrm{x}$ \\
\hline 7. & $\begin{array}{l}\text { Allama Iqbal } \\
\text { Open } \\
\text { University }\end{array}$ & 5 & 5 & 6 & 4 or 1 & $\mathrm{x}$ & $\mathrm{x}$ \\
\hline 8. & $\begin{array}{l}\text { Sargodha } \\
\text { University* }\end{array}$ & 6 & 6 & 6 & 6 & $\mathrm{x}$ & $\mathrm{x}$ \\
\hline 9. & $\begin{array}{l}\text { Khushal Khan } \\
\text { Khattak } \\
\text { University }\end{array}$ & 6 & 6 & 6 & 4 or 5 & $\mathrm{x}$ & $\mathrm{x}$ \\
\hline
\end{tabular}


*BS Program of 8 Semesters divided as follows:

\begin{tabular}{|c|l|c|c|c|c|c|c|c|c|}
\hline S.No & NAME OF UNIVERSITY & \multicolumn{10}{|c|}{ SEMESTERS } \\
\cline { 3 - 10 } & & $\mathbf{1}^{\text {st }}$ & $\mathbf{2}^{\text {nd }}$ & $\mathbf{3}^{\text {rd }}$ & $\mathbf{4}^{\text {th }}$ & $\mathbf{5}^{\text {th }}$ & $\mathbf{6}^{\text {th }}$ & $\mathbf{7}^{\text {th }}$ & $\mathbf{8}^{\text {th }}$ \\
\hline 1. & University of Sindh & 6 & 6 & 5 & 5 & 5 & 5 & 6 & 5 \\
\hline 2. & $\begin{array}{l}\text { Islamia University } \\
\text { Bahawalpur }\end{array}$ & 6 & 6 & 5 & 5 & 5 & 6 & 6 & 4 \\
\hline 3. & Sargodha University & 6 & 6 & 5 & 5 & 5 & 5 & 6 & 6 \\
\hline 4. & $\begin{array}{l}\text { Khushal Khan Khattak } \\
\text { Univ. }\end{array}$ & 6 & 6 & 5 & 5 & 5 & 5 & 6 & 4 \\
\hline
\end{tabular}

Karachi University teaches 27 courses in all in 2 years or 4 semesters (13 in BLIS and 14 in MLIS), Islamia University and Sargodha University each teaches 24 courses in all in 2-year program and 43,44 courses respectively in 4-year program. Khushal Khan Khattak University offers 22 or 23 courses in MLIS and 42 courses in 4-year program. The students of Punjab University have to study 25 courses, besides 8-week mandatory non-credit practicum (internship) in 2 years to get Masters degree in Information Management. Fulfillment of 20 courses (without thesis) or 17 courses (in case of research thesis) is the requirement of Allama Iqbal Open University. University of Sindh is unique in a sense that it is the only library school which offers only 4-year program and students are required to study 43 courses in all to complete their program. Peshawar University offers 12 courses (under annual system) whereas Baluchistan University teaches 20 courses to complete 2-year Masters program.

\section{Courses offered at MA (Previous)/BLIS (or equivalent) Level}

At present only University of Karachi is offering one-year postgraduate BLIS program and after qualification, the graduates are eligible to further study one-year MLIS program. The BLIS program of University of Karachi is equivalent to M.A. (Previous) of all other universities of Pakistan (except Allama Iqbal Open University, where degree of BLIS is equivalent to B.A., B.Sc., B.Com. etc). The courses offered at this level are the core courses and the students have to study all the courses without any choice or option. The following table shows the maximum titles of the courses being offered at BLIS/MA (Previous) or equivalent level by the universities of Pakistan: 
Table-4 Titles of the courses offered at BLIS or equivalent level

\begin{tabular}{|c|c|c|c|c|c|c|c|c|c|c|c|}
\hline $\begin{array}{l}\text { S.N } \\
\text { o }\end{array}$ & Course Title & $\begin{array}{l}\mathbf{K} \\
\mathbf{U}\end{array}$ & $\begin{array}{l}\mathbf{P u} \\
\mathbf{U}\end{array}$ & $\begin{array}{l}\mathbf{S i} \\
\mathbf{U}\end{array}$ & $\begin{array}{l}\mathbf{P e} \\
\mathbf{U}\end{array}$ & $\begin{array}{l}\mathbf{B} \\
\mathbf{U}\end{array}$ & $\begin{array}{l}\mathbf{I} \\
\mathbf{U}\end{array}$ & $\begin{array}{l}\mathbf{A} \\
\mathbf{U}\end{array}$ & $\begin{array}{l}\text { Sa } \\
\text { U }\end{array}$ & KK & $\begin{array}{l}\text { Tot } \\
\text { al }\end{array}$ \\
\hline 1. & $\begin{array}{l}\text { Bibliography \& Bib. } \\
\text { Control }\end{array}$ & $\mathrm{Y}$ & $\mathrm{N}$ & $\mathrm{N}$ & $\mathrm{Y}$ & $\mathrm{N}$ & $\mathrm{Y}$ & $\mathrm{N}$ & $\mathrm{Y}$ & $\mathrm{N}$ & 4 \\
\hline 2. & $\begin{array}{l}\text { Cataloguing } \\
\text { (Practical) }\end{array}$ & $\mathrm{Y}$ & $\mathrm{Y}$ & $\mathrm{Y}$ & $\mathrm{Y}$ & $\mathrm{Y}$ & $\mathrm{Y}$ & $Y$ & $\mathrm{Y}$ & $\mathrm{Y}$ & 9 \\
\hline 3. & Cataloguing (Theory) & $\mathrm{Y}$ & $\mathrm{N}$ & $\mathrm{N}$ & $\mathrm{Y}$ & $\mathrm{N}$ & $\mathrm{N}$ & $Y$ & $\mathrm{Y}$ & $\mathrm{Y}$ & 5 \\
\hline 4. & $\begin{array}{l}\text { Classification } \\
\text { (Practical) }\end{array}$ & $\mathrm{Y}$ & $\mathrm{Y}$ & $\mathrm{Y}$ & $\mathrm{Y}$ & $\mathrm{Y}$ & $\mathrm{Y}$ & $Y$ & Y & $\mathrm{Y}$ & 9 \\
\hline 5. & $\begin{array}{l}\text { Classification } \\
\text { (Theory) }\end{array}$ & $\mathrm{Y}$ & $\mathrm{N}$ & $\mathrm{N}$ & $\mathrm{Y}$ & $\mathrm{N}$ & $\mathrm{N}$ & $Y$ & $\mathrm{Y}$ & $\mathrm{Y}$ & 5 \\
\hline 6. & $\begin{array}{l}\text { Collection Dev. \& } \\
\text { Management }\end{array}$ & $\mathrm{Y}$ & $\mathrm{N}$ & $\mathrm{Y}$ & $\mathrm{Y}$ & $\mathrm{Y}$ & $\mathrm{Y}$ & $\mathrm{Y}$ & $\mathrm{Y}$ & $\mathrm{Y}$ & 8 \\
\hline 7. & Communication Skills & $\mathrm{Y}$ & $\mathrm{Y}$ & $\mathrm{N}$ & $\mathrm{N}$ & $\mathrm{N}$ & $\mathrm{N}$ & $\mathrm{N}$ & $\mathrm{N}$ & $Y$ & 3 \\
\hline 8. & $\begin{array}{l}\text { Foundation of Inf. } \\
\text { Based Org. }\end{array}$ & $\mathrm{N}$ & $\bar{Y}$ & $\mathrm{~N}$ & $\mathrm{~N}$ & $\mathrm{~N}$ & $\mathrm{~N}$ & $\mathrm{~N}$ & $\mathrm{~N}$ & $\mathrm{~N}$ & 1 \\
\hline 9. & $\begin{array}{l}\text { Foundation of } \\
\text { Librarianship }\end{array}$ & $\mathrm{N}$ & $\mathrm{N}$ & $\mathrm{N}$ & $\mathrm{N}$ & $\mathrm{N}$ & $\mathrm{Y}$ & $\mathrm{Y}$ & $\mathrm{Y}$ & $\mathrm{N}$ & 3 \\
\hline 10. & $\begin{array}{l}\text { Fundamentals of } \\
\text { Management }\end{array}$ & $\mathrm{N}$ & $\mathrm{N}$ & $\mathrm{N}$ & $\mathrm{N}$ & $\mathrm{N}$ & $\mathrm{N}$ & $\mathrm{N}$ & $\mathrm{N}$ & $Y$ & 1 \\
\hline 11. & $\begin{array}{l}\text { History of Books \& } \\
\text { Libraries }\end{array}$ & $\mathrm{Y}$ & $\mathrm{N}$ & $\mathrm{N}$ & $\mathrm{N}$ & $\mathrm{N}$ & $\mathrm{N}$ & $\mathrm{N}$ & $\mathrm{N}$ & $\mathrm{N}$ & 1 \\
\hline 12. & $\begin{array}{l}\text { Human Information } \\
\text { Behavior }\end{array}$ & $\mathrm{N}$ & $\mathrm{Y}$ & $\mathrm{N}$ & $\mathrm{N}$ & $\mathrm{N}$ & $\mathrm{N}$ & $\mathrm{N}$ & $\mathrm{N}$ & $\mathrm{N}$ & 1 \\
\hline 13. & $\begin{array}{l}\text { Information Literacy } \\
\text { Instruction }\end{array}$ & $\mathrm{N}$ & $\bar{Y}$ & $\mathrm{Y}$ & $\mathrm{N}$ & $\mathrm{Y}$ & $\mathrm{N}$ & $\mathrm{N}$ & $\mathrm{Y}$ & $\mathrm{N}$ & 4 \\
\hline 14. & $\begin{array}{l}\text { Int. to Publishing \& } \\
\text { Book Trade }\end{array}$ & $\mathrm{N}$ & $\mathrm{N}$ & $\mathrm{Y}$ & $\mathrm{N}$ & $\mathrm{Y}$ & $\mathrm{N}$ & $\mathrm{N}$ & $\mathrm{N}$ & $\mathrm{N}$ & 2 \\
\hline 15. & Introduction to IT & $\mathrm{N}$ & $\mathrm{N}$ & $\mathrm{N}$ & $\mathrm{N}$ & $\mathrm{N}$ & $\mathrm{N}$ & $\mathrm{N}$ & $\mathrm{N}$ & $\mathrm{Y}$ & 1 \\
\hline 16. & Introduction to LIS & $\mathrm{N}$ & $\mathrm{N}$ & $\mathrm{N}$ & $\mathrm{Y}$ & $\mathrm{N}$ & $\mathrm{N}$ & $\mathrm{Y}$ & $\mathrm{N}$ & $\mathrm{N}$ & 2 \\
\hline 17. & $\begin{array}{l}\text { IT: Concepts and } \\
\text { Application }\end{array}$ & $\mathrm{N}$ & $\mathrm{Y}$ & $\mathrm{N}$ & $\mathrm{N}$ & $\mathrm{N}$ & $\mathrm{Y}$ & $\mathrm{N}$ & $\mathrm{Y}$ & $\mathrm{Y}$ & 4 \\
\hline 18. & Library \& Society & $\mathrm{Y}$ & $\mathrm{N}$ & $\mathrm{N}$ & $\mathrm{N}$ & $\mathrm{N}$ & $\mathrm{N}$ & $\mathrm{N}$ & $\mathrm{N}$ & $\mathrm{Y}$ & 2 \\
\hline 19. & $\begin{array}{l}\text { Library } \\
\text { Automation/IS\&R* }\end{array}$ & $\mathrm{N}$ & $\mathrm{N}$ & $\mathrm{Y}$ & $\mathrm{N}$ & $\mathrm{Y}$ & $\mathrm{Y}$ & $\mathrm{Y}$ & $\mathrm{N}$ & $\mathrm{N}$ & 4 \\
\hline 20. & $\begin{array}{l}\text { Library } \\
\text { Correspondence }\end{array}$ & $\mathrm{Y}$ & $\mathrm{N}$ & $\mathrm{N}$ & $\mathrm{N}$ & $\mathrm{N}$ & $\mathrm{N}$ & $\mathrm{N}$ & $\mathrm{N}$ & $\mathrm{N}$ & 1 \\
\hline 21. & Library Operation & $\mathrm{Y}$ & $\mathrm{N}$ & $\mathrm{N}$ & $\mathrm{N}$ & $\mathrm{N}$ & $\mathrm{N}$ & $\mathrm{N}$ & $\mathrm{N}$ & $\mathrm{N}$ & 1 \\
\hline 22. & Literature on Islam & $\mathrm{N}$ & $\mathrm{N}$ & $\mathrm{N}$ & $\mathrm{N}$ & $\mathrm{N}$ & $\mathrm{Y}$ & $\mathrm{N}$ & $\mathrm{N}$ & $\mathrm{N}$ & 1 \\
\hline 23. & Management\& Admn. & $\mathrm{Y}$ & $\mathrm{Y}$ & $\mathrm{Y}$ & $\mathrm{Y}$ & $\mathrm{Y}$ & $\mathrm{Y}$ & $\mathrm{Y}$ & $\mathrm{Y}$ & $\mathrm{Y}$ & 9 \\
\hline
\end{tabular}




\begin{tabular}{|c|c|c|c|c|c|c|c|c|c|c|c|}
\hline & of LIC** & & & & & & & & & & \\
\hline 24. & $\begin{array}{l}\text { Managing Collection \& } \\
\text { Online }\end{array}$ & $\mathrm{N}$ & $\mathrm{Y}$ & $\mathrm{N}$ & $\mathrm{N}$ & $\mathrm{N}$ & $\mathrm{N}$ & $\mathrm{N}$ & $\mathrm{N}$ & $\mathrm{N}$ & 1 \\
\hline 25. & $\begin{array}{l}\text { Marketing of Lib \& Inf } \\
\text { Services }\end{array}$ & $\mathrm{N}$ & $\mathrm{N}$ & $\mathrm{Y}$ & $\mathrm{N}$ & $\mathrm{Y}$ & $\mathrm{N}$ & $\mathrm{N}$ & $\mathrm{N}$ & $\mathrm{N}$ & 2 \\
\hline 26. & $\begin{array}{l}\text { Online Information } \\
\text { Retrieval }\end{array}$ & $\mathrm{N}$ & $\mathrm{Y}$ & $\mathrm{N}$ & $\mathrm{N}$ & $\mathrm{N}$ & $\mathrm{N}$ & $\mathrm{N}$ & $Y$ & $\mathrm{~N}$ & 2 \\
\hline 27. & $\begin{array}{l}\text { Organization of } \\
\text { Information }\end{array}$ & $\mathrm{N}$ & $\mathrm{Y}$ & $\mathrm{N}$ & $\mathrm{N}$ & $\mathrm{N}$ & $Y$ & $\mathrm{~N}$ & $\mathrm{~N}$ & $\mathrm{~N}$ & 2 \\
\hline 28. & $\begin{array}{l}\text { Personality Dev. \& } \\
\text { Com. Skills }\end{array}$ & $\mathrm{N}$ & $\mathrm{Y}$ & $\mathrm{N}$ & $\mathrm{N}$ & $\mathrm{N}$ & $\mathrm{Y}$ & $\mathrm{N}$ & $\mathrm{N}$ & $\mathrm{N}$ & 2 \\
\hline 29. & $\begin{array}{l}\text { Qualitative Research } \\
\text { Methods }\end{array}$ & $\mathrm{N}$ & $\mathrm{N}$ & $Y$ & $\mathrm{~N}$ & $\mathrm{~N}$ & $\mathrm{~N}$ & $\mathrm{~N}$ & $\mathrm{~N}$ & $\mathrm{~N}$ & 1 \\
\hline 30. & $\begin{array}{l}\text { Quantitative Research } \\
\text { Methods }\end{array}$ & $\mathrm{N}$ & $\mathrm{N}$ & $\mathrm{N}$ & $\mathrm{N}$ & $\bar{Y}$ & $\mathrm{~N}$ & $\mathrm{~N}$ & $\mathrm{~N}$ & $\mathrm{~N}$ & 1 \\
\hline 31. & $\begin{array}{l}\text { Ref./Inf. Sources \& } \\
\text { Services }\end{array}$ & $\mathrm{Y}$ & $\mathrm{Y}$ & $\mathrm{N}$ & $\mathrm{N}$ & $\mathrm{N}$ & $Y$ & $\mathrm{Y}$ & $Y$ & $\mathrm{Y}$ & 6 \\
\hline 32. & $\begin{array}{l}\text { Research } \\
\text { Methodology }\end{array}$ & $\mathrm{N}$ & $\mathrm{N}$ & $Y$ & $\mathrm{~N}$ & $\mathrm{Y}$ & $\mathrm{N}$ & $\mathrm{N}$ & $\mathrm{N}$ & $\mathrm{N}$ & 2 \\
\hline 33. & Serial Management & $\mathrm{N}$ & $\mathrm{N}$ & $\mathrm{N}$ & $\mathrm{Y}$ & $\mathrm{N}$ & $\mathrm{N}$ & $\mathrm{N}$ & $\mathrm{N}$ & $\mathrm{N}$ & 1 \\
\hline & Total .............. & $\begin{array}{l}1 \\
3\end{array}$ & 13 & $\begin{array}{l}\mathbf{1} \\
\mathbf{0}\end{array}$ & 9 & $\begin{array}{l}\mathbf{1} \\
\mathbf{0}\end{array}$ & $\begin{array}{l}1 \\
2\end{array}$ & $\begin{array}{l}\mathbf{1} \\
\mathbf{0}\end{array}$ & 12 & 12 & 101 \\
\hline
\end{tabular}

*IS\&R=Information Storage \& Retrieval Centers

Key:

KU = Karachi University, Karachi

PuU = Punjab University, Lahore

SiU = Sindh University, Jamshoro

PeU = Peshawar University, Peshawar

BU = Baluchistan University, Quetta

IU= Islamia University, Bahawalpur

AU = Allama Iqbal Open University, Isl

SaU = Sargodha University, Sargodha

KK = Khushal Khan Khattak University, Karak

The above 33 courses (in all) are being offered at M.A (Previous) or equivalent level by the nine library schools of Pakistan. Some of the core courses are common and are taught in majority of the library schools but most of the courses are taught independently or in two to three library schools. The fundamental core courses like Classification, Cataloguing, and Management and Administration of Library and Information Centers are being offered by all nine library schools. The course of Collection Development is not offered by Punjab University and the course of 
Reference and Information Sources and Services is not offered by Sindh University, Peshawar University, and Baluchistan University whereas remaining six universities are offering this important core course. Sindh University (which is practicing 4-year BS program) offers this course under the title "Basic Reference Sources" in fourth semester. Although the table shows that theory of Cataloguing and theory of Classification are not offered by Punjab University, Sindh University, and Islamia University but fact is that Punjab University and Islamia University are offering these courses under the title "Organization of Information" whereas Sindh University is offering these courses under single title "Organization of Information" in fourth semester.

\section{Courses offered at MA (Final)/MLIS Level}

Only University of Karachi is offering two separate postgraduate programs of BLIS and MLIS of one year each. These programs are equivalent to M.A. (Previous) and M.A. (Final) of other universities of Pakistan. The courses offered in first year have been discussed in the above table, whereas courses of second year are being enumerated here. The following table shows the maximum titles of the courses being offered by the universities of Pakistan at MLIS/M.A. (Final) level:

Table-5 Titles of the courses offered at MLIS or equivalent level

\begin{tabular}{|c|l|c|c|c|c|c|c|c|c|c|c|}
\hline S.No. & Course Title & KU & PuU & SiU & PeU & BU & IU & AU & SaU & KK & Total \\
\hline 1. & $\begin{array}{l}\text { Academic } \\
\text { Libraries }\end{array}$ & $\mathrm{Y}^{*}$ & $\mathrm{~N}$ & $\mathrm{~N}$ & $\mathrm{Y}^{*}$ & $\mathrm{~N}$ & $\mathrm{Y}^{*}$ & $\mathrm{~N}$ & $\mathrm{Y}^{*}$ & $\mathrm{~N}$ & $\mathbf{4}$ \\
\hline 2. & $\begin{array}{l}\text { Advanced } \\
\text { Management } \\
\text { Techniques \& } \\
\text { Operations }\end{array}$ & $\mathrm{N}$ & $\mathrm{N}$ & $\mathrm{N}$ & $\mathrm{N}$ & $\mathrm{N}$ & $\mathrm{Y}^{*}$ & $\mathrm{~N}$ & $\mathrm{Y}^{*}$ & $\mathrm{~N}$ & $\mathbf{2}$ \\
\hline 3 & $\begin{array}{l}\text { Advanced } \\
\text { Management \& } \\
\text { Leadership Skills }\end{array}$ & $\mathrm{N}$ & $\mathrm{Y}^{*}$ & $\mathrm{~N}$ & $\mathrm{~N}$ & $\mathrm{~N}$ & $\mathrm{~N}$ & $\mathrm{~N}$ & $\mathrm{~N}$ & $\mathrm{~N}$ & $\mathbf{1}$ \\
\hline 4. & $\begin{array}{l}\text { Adv. Tech. } \\
\text { Operations/Adv. } \\
\text { Cataloguing \& } \\
\text { Classification }\end{array}$ & $\mathrm{N}$ & $\mathrm{Y}^{*}$ & $\mathrm{~N}$ & $\mathrm{Y}$ & $\mathrm{N}$ & $\mathrm{Y}^{*}$ & $\mathrm{Y} 2$ & $\mathrm{Y}^{*}$ & $\mathrm{~N}$ & $\mathbf{6}$ \\
\hline 5. & $\begin{array}{l}\text { Agricultural } \\
\text { Libraries }\end{array}$ & $\mathrm{N}$ & $\mathrm{N}$ & $\mathrm{N}$ & $\mathrm{N}$ & $\mathrm{N}$ & $\mathrm{Y}^{*}$ & $\mathrm{~N}$ & $\mathrm{Y}^{*}$ & $\mathrm{~N}$ & $\mathbf{2}$ \\
\hline 6. & $\begin{array}{l}\text { Application of } \\
\text { Information } \\
\text { Systems }\end{array}$ & $\mathrm{N}$ & $\mathrm{Y}^{*}$ & $\mathrm{~N}$ & $\mathrm{~N}$ & $\mathrm{~N}$ & $\mathrm{~N}$ & $\mathrm{~N}$ & $\mathrm{~N}$ & $\mathrm{~N}$ & $\mathbf{1}$ \\
\hline 7. & $\begin{array}{l}\text { Archives \& } \\
\text { Record }\end{array}$ & $\mathrm{Y}^{*}$ & $\mathrm{Y}$ & $\mathrm{Y}^{*}$ & $\mathrm{Y}^{*}$ & $\mathrm{Y}^{*}$ & $\mathrm{Y}^{*}$ & $\mathrm{~N}$ & $\mathrm{Y}^{*}$ & $\mathrm{Y}$ & $\mathbf{8}$ \\
\hline
\end{tabular}


Content Analysis of Library \& Information Science (LIS) Curricula in Pakistani Universities

\begin{tabular}{|c|c|c|c|c|c|c|c|c|c|c|c|}
\hline & Management & & & & & & & & & & \\
\hline 8. & $\begin{array}{l}\text { Basic Research } \\
\text { Methods \& } \\
\text { Techniques } \\
\end{array}$ & $\mathrm{N}$ & $\mathrm{N}$ & $\mathrm{N}$ & $\mathrm{N}$ & $\mathrm{N}$ & $\mathrm{N}$ & $\mathrm{N}$ & $\mathrm{N}$ & $\mathrm{Y}$ & 1 \\
\hline 9. & $\begin{array}{l}\text { Bibliographical } \\
\text { Control }\end{array}$ & $\mathrm{N}$ & $\mathrm{N}$ & $\mathrm{Y}^{*}$ & $\mathrm{~N}$ & $\mathrm{Y}^{*}$ & $\mathrm{~N}$ & $\mathrm{~N}$ & $\mathrm{~N}$ & $\mathrm{Y}^{*}$ & 3 \\
\hline 10. & $\begin{array}{l}\text { Business \& } \\
\text { Industry } \\
\text { Information }\end{array}$ & $\mathrm{N}$ & $\mathrm{N}$ & $\mathrm{N}$ & $\mathrm{N}$ & $\mathrm{Y}^{*}$ & $\mathrm{~N}$ & $\mathrm{~N}$ & $\mathrm{~N}$ & $\mathrm{~N}$ & 1 \\
\hline 11. & $\begin{array}{l}\text { Cataloguing of } \\
\text { Special Material }\end{array}$ & $\mathrm{Y}$ & $\mathrm{N}$ & $\mathrm{N}$ & $\mathrm{N}$ & $\mathrm{N}$ & $\mathrm{N}$ & $\mathrm{N}$ & $\mathrm{N}$ & $\mathrm{N}$ & 1 \\
\hline 12. & $\begin{array}{l}\text { Children } \\
\text { Libraries }\end{array}$ & $\mathrm{N}$ & $\mathrm{N}$ & $\mathrm{N}$ & $\mathrm{N}$ & $\mathrm{N}$ & $\mathrm{Y}^{*}$ & $\mathrm{~N}$ & $\mathrm{Y}^{*}$ & $\mathrm{~N}$ & 2 \\
\hline 13. & $\begin{array}{l}\text { Comparative \& } \\
\text { Int Librarianship }\end{array}$ & $\mathrm{Y}$ & $\mathrm{N}$ & $\mathrm{N}$ & $\mathrm{N}$ & $\mathrm{N}$ & $\mathrm{Y}^{*}$ & $\mathrm{~N}$ & $\mathrm{Y}^{*}$ & $\mathrm{~N}$ & 3 \\
\hline 14. & $\begin{array}{l}\text { Comparative } \\
\text { Classification }\end{array}$ & $\mathrm{Y}$ & $\mathrm{N}$ & $\mathrm{N}$ & $\mathrm{N}$ & $\mathrm{N}$ & $\mathrm{N}$ & $\mathrm{N}$ & $\mathrm{N}$ & $\mathrm{N}$ & 1 \\
\hline 15. & $\begin{array}{l}\text { Computer } \\
\text { Networks }\end{array}$ & $\mathrm{N}$ & $\mathrm{N}$ & $\mathrm{N}$ & $\mathrm{N}$ & $\mathrm{N}$ & $\mathrm{Y}^{*}$ & $\mathrm{~N}$ & $\mathrm{Y}^{*}$ & $\mathrm{~N}$ & 2 \\
\hline 16. & $\begin{array}{l}\text { Computer } \\
\text { Programmng for } \\
\text { I.M^ }^{\wedge}\end{array}$ & $\mathrm{N}$ & $\mathrm{N}$ & $\mathrm{N}$ & $\mathrm{N}$ & $\mathrm{N}$ & $\mathrm{Y}^{*}$ & $\mathrm{~N}$ & $\mathrm{Y}^{*}$ & $\mathrm{~N}$ & 2 \\
\hline 17. & $\begin{array}{l}\text { Database Design } \\
\text { \& Management }\end{array}$ & $\mathrm{N}$ & $\mathrm{N}$ & $\mathrm{Y}^{*}$ & $\mathrm{~N}$ & $\mathrm{Y}^{*}$ & $\mathrm{Y}^{*}$ & $\mathrm{~N}$ & $\mathrm{Y}^{*}$ & $\mathrm{~N}$ & 4 \\
\hline 18. & Digital Library & $\mathrm{Y}$ & $\mathrm{Y}^{*}$ & $\mathrm{Y}^{*}$ & $\mathrm{~N}$ & $\mathrm{Y}^{*}$ & $\mathrm{Y}^{*}$ & $\mathrm{~N}$ & $\mathrm{Y}^{*}$ & $\mathrm{Y}$ & 7 \\
\hline 19. & $\begin{array}{l}\text { Electronic } \\
\text { Collection } \\
\text { /Resources } \\
\text { Management }\end{array}$ & $\mathrm{N}$ & $\mathrm{Y}^{*}$ & $\mathrm{~N}$ & $\mathrm{~N}$ & $\mathrm{~N}$ & $\mathrm{Y}^{*}$ & $\mathrm{~N}$ & $\mathrm{~N}$ & $\mathrm{~N}$ & 2 \\
\hline 20. & $\begin{array}{l}\text { Financial } \\
\text { Management }\end{array}$ & $\mathrm{N}$ & $\mathrm{N}$ & $\mathrm{Y}^{*}$ & $\mathrm{~N}$ & $\mathrm{Y}^{*}$ & $\mathrm{Y}^{*}$ & $\mathrm{~N}$ & $\mathrm{Y}^{*}$ & $\mathrm{~N}$ & 4 \\
\hline 21. & $\begin{array}{l}\text { Health \& Medical } \\
\text { Sc. Libraries }\end{array}$ & $\mathrm{Y}^{*}$ & $\mathrm{~N}$ & $\mathrm{~N}$ & $\mathrm{~N}$ & $\mathrm{~N}$ & $\mathrm{Y}^{*}$ & $\mathrm{~N}$ & $\mathrm{Y}^{*}$ & $\mathrm{~N}$ & 3 \\
\hline 22. & $\begin{array}{l}\text { Health Sciences } \\
\text { Information }\end{array}$ & $\mathrm{N}$ & $\mathrm{N}$ & $\mathrm{N}$ & $\mathrm{N}$ & $\mathrm{Y}^{*}$ & $\mathrm{~N}$ & $\mathrm{~N}$ & $\mathrm{~N}$ & $\mathrm{~N}$ & 1 \\
\hline 23. & $\begin{array}{l}\text { Human Resource } \\
\text { Management }\end{array}$ & $\mathrm{N}$ & $\mathrm{N}$ & $\mathrm{Y}^{*}$ & $\mathrm{~N}$ & $\mathrm{Y}^{*}$ & $\mathrm{Y}^{*}$ & $\mathrm{~N}$ & $\mathrm{Y}^{*}$ & $\mathrm{~N}$ & 4 \\
\hline 24. & $\begin{array}{l}\text { Humanities \& } \\
\text { Soc. Sc. Info. }\end{array}$ & $\mathrm{N}$ & $\mathrm{Y}^{*}$ & $\mathrm{~N}$ & $\mathrm{~N}$ & $\mathrm{~N}$ & $\mathrm{~N}$ & $\mathrm{~N}$ & $\mathrm{~N}$ & $\mathrm{~N}$ & 1 \\
\hline 25. & $\begin{array}{l}\text { Indexing and } \\
\text { Abstracting }\end{array}$ & $\mathrm{Y}$ & $\mathrm{Y}^{*}$ & $\mathrm{Y}$ & $\mathrm{N}$ & $\mathrm{Y}$ & $\mathrm{Y}^{*}$ & $\mathrm{~N}$ & $\mathrm{Y}^{*}$ & $\mathrm{Y}$ & 7 \\
\hline 26. & Information & $\mathrm{N}$ & $\mathrm{N}$ & $\mathrm{N}$ & $\mathrm{N}$ & $\mathrm{N}$ & $\mathrm{Y}^{*}$ & $\mathrm{~N}$ & $\mathrm{~N}$ & $\mathrm{~N}$ & 1 \\
\hline
\end{tabular}


Jhss, Vol. 9, No. 1, January to June, 2018

\begin{tabular}{|c|c|c|c|c|c|c|c|c|c|c|c|}
\hline & Architecture & & & & & & & & & & \\
\hline 27. & $\begin{array}{l}\text { Information } \\
\text { Literacy } \\
\text { Instruction }\end{array}$ & $\mathrm{N}$ & $\mathrm{N}$ & $\mathrm{N}$ & $\mathrm{N}$ & $\mathrm{N}$ & $\mathrm{Y}^{*}$ & $\mathrm{~N}$ & $\mathrm{~N}$ & $\mathrm{~N}$ & 1 \\
\hline 28. & $\begin{array}{l}\text { Information } \\
\text { Policy }\end{array}$ & $\mathrm{N}$ & $\mathrm{N}$ & $\mathrm{N}$ & $\mathrm{N}$ & $\mathrm{N}$ & $\mathrm{Y}^{*}$ & $\mathrm{~N}$ & $\mathrm{Y}^{*}$ & $\mathrm{~N}$ & 2 \\
\hline 29. & $\begin{array}{l}\text { Information } \\
\text { Seeking \& Use }\end{array}$ & $\mathrm{N}$ & $\mathrm{N}$ & $\mathrm{N}$ & $\mathrm{N}$ & $\mathrm{N}$ & $\mathrm{Y}^{*}$ & $\mathrm{~N}$ & $\mathrm{Y}^{*}$ & $\mathrm{Y}^{*}$ & 3 \\
\hline 30. & $\begin{array}{l}\text { Information } \\
\text { Sources and Lit. } \\
* *\end{array}$ & $\mathrm{Y}^{*}$ & $\mathrm{Y}^{*}$ & $\mathrm{Y}^{*}$ & $\mathrm{Y}^{*}$ & $\mathrm{Y}^{*}$ & $\mathrm{Y}^{*}$ & $\mathrm{~N}$ & $\mathrm{Y}^{*}$ & $\mathrm{Y}^{*}$ & 8 \\
\hline 31. & $\begin{array}{l}\text { Information } \\
\text { Storage \& } \\
\text { Retrieval } \\
\end{array}$ & $\mathrm{Y}$ & $\mathrm{N}$ & $\mathrm{Y}$ & $\mathrm{N}$ & $\mathrm{Y}$ & $\mathrm{N}$ & $\mathrm{N}$ & $\mathrm{N}$ & $\mathrm{Y}$ & 4 \\
\hline 32. & $\begin{array}{l}\text { Intellectual } \\
\text { Property Rights }\end{array}$ & $\mathrm{N}$ & $\mathrm{N}$ & $\mathrm{Y}^{*}$ & $\mathrm{~N}$ & $\mathrm{Y}^{*}$ & $\mathrm{~N}$ & $\mathrm{~N}$ & $\mathrm{~N}$ & $\mathrm{~N}$ & 2 \\
\hline 33. & Internship & $\mathrm{N}$ & $\mathrm{N}$ & $\mathrm{Y}$ & $\mathrm{N}$ & $\mathrm{N}$ & $\mathrm{N}$ & $\mathrm{N}$ & $\mathrm{N}$ & $\mathrm{N}$ & 1 \\
\hline 34. & $\begin{array}{l}\text { Introduction to } \\
\text { Computers }\end{array}$ & $\mathrm{N}$ & $\mathrm{N}$ & $\mathrm{N}$ & $\mathrm{Y}^{*}$ & $\mathrm{~N}$ & $\mathrm{~N}$ & $\mathrm{~N}$ & $\mathrm{~N}$ & $\mathrm{~N}$ & 1 \\
\hline 35. & IT and Libraries & $\mathrm{Y}$ & $\mathrm{N}$ & $\mathrm{N}$ & $\mathrm{Y}$ & $\mathrm{N}$ & $\mathrm{N}$ & $\mathrm{N}$ & $\mathrm{N}$ & $\mathrm{N}$ & 2 \\
\hline 36. & $\begin{array}{l}\text { Knowledge } \\
\text { Management }\end{array}$ & $\mathrm{N}$ & $\mathrm{Y}$ & $\mathrm{Y}^{*}$ & $\mathrm{~N}$ & $\mathrm{Y}^{*}$ & $\mathrm{Y}^{*}$ & $\mathrm{~N}$ & $\mathrm{Y}^{*}$ & $\mathrm{Y}$ & 6 \\
\hline 37. & Law Libraries & $\mathrm{N}$ & $\mathrm{N}$ & $\mathrm{N}$ & $\mathrm{N}$ & $\mathrm{N}$ & $\mathrm{Y}^{*}$ & $\mathrm{~N}$ & $\mathrm{Y}^{*}$ & $\mathrm{~N}$ & 2 \\
\hline 38. & $\begin{array}{l}\text { Lib. \& Inf. } \\
\text { Services in } \\
\text { Pakistan } \\
\end{array}$ & $\mathrm{N}$ & $\mathrm{N}$ & $\mathrm{Y}$ & $\mathrm{N}$ & $\mathrm{Y}$ & $\mathrm{N}$ & $\mathrm{N}$ & $\mathrm{N}$ & $\mathrm{N}$ & 2 \\
\hline 39. & $\begin{array}{l}\text { Lib. \& Inf. } \\
\text { Software } \\
\text { Packages }\end{array}$ & $\mathrm{N}$ & $\mathrm{N}$ & $\mathrm{N}$ & $\mathrm{N}$ & $\mathrm{N}$ & $\mathrm{N}$ & $\mathrm{N}$ & $\mathrm{N}$ & $\mathrm{Y}^{*}$ & 1 \\
\hline 40. & $\begin{array}{l}\text { Libraries and } \\
\text { Society }\end{array}$ & $\mathrm{N}$ & $\mathrm{N}$ & $\mathrm{N}$ & $\mathrm{N}$ & $\mathrm{N}$ & $\mathrm{Y}$ & $\mathrm{N}$ & $\mathrm{Y}^{*}$ & $\mathrm{~N}$ & 2 \\
\hline 41. & $\begin{array}{l}\text { Library } \\
\text { Automation } \\
\text { Systems } \\
\end{array}$ & $\mathrm{N}$ & $\mathrm{Y}$ & $\mathrm{N}$ & $\mathrm{N}$ & $\mathrm{N}$ & $\mathrm{N}$ & $\mathrm{N}$ & $\mathrm{Y}$ & $\mathrm{N}$ & 2 \\
\hline 42. & $\begin{array}{l}\text { Management of } \\
\text { Info. Systems }\end{array}$ & $\mathrm{N}$ & $\mathrm{N}$ & $\mathrm{N}$ & $\mathrm{Y}^{*}$ & $\mathrm{~N}$ & $\mathrm{~N}$ & $\mathrm{~N}$ & $\mathrm{~N}$ & $\mathrm{~N}$ & 1 \\
\hline 43. & $\begin{array}{l}\text { Marketing of Lib. } \\
\text { Info. Services }\end{array}$ & $\mathrm{N}$ & $\mathrm{Y}$ & $\mathrm{N}$ & $\mathrm{Y}^{*}$ & $\mathrm{~N}$ & $\mathrm{Y}$ & $\mathrm{N}$ & $Y$ & $\mathrm{Y}$ & 5 \\
\hline 44. & $\begin{array}{l}\text { Media } \\
\text { Information } \\
\text { Management } \\
\end{array}$ & $\mathrm{N}$ & $\mathrm{Y}^{*}$ & $\mathrm{~N}$ & $\mathrm{~N}$ & $\mathrm{~N}$ & $\mathrm{~N}$ & $\mathrm{~N}$ & $\mathrm{~N}$ & $\mathrm{~N}$ & 1 \\
\hline 45. & $\begin{array}{l}\text { Media } \\
\text { Librarianship }\end{array}$ & $\mathrm{N}$ & $\mathrm{N}$ & $\mathrm{Y}^{*}$ & $\mathrm{Y}^{*}$ & $\mathrm{Y}^{*}$ & $\mathrm{Y}^{*}$ & $\mathrm{~N}$ & $\mathrm{Y}^{*}$ & $\mathrm{~N}$ & 5 \\
\hline
\end{tabular}


Content Analysis of Library \& Information Science (LIS) Curricula in Pakistani Universities

\begin{tabular}{|c|c|c|c|c|c|c|c|c|c|c|c|}
\hline 46. & $\begin{array}{l}\text { Media } \\
\text { Technology \& } \\
\text { Resources }\end{array}$ & $\mathrm{Y}^{*}$ & $\mathrm{~N}$ & $\mathrm{~N}$ & $\mathrm{~N}$ & $\mathrm{~N}$ & $\mathrm{~N}$ & $\mathrm{~N}$ & $\mathrm{~N}$ & $\mathrm{~N}$ & 1 \\
\hline 47. & $\begin{array}{l}\text { Models for } \\
\text { Lib.Management, } \\
\text { Decision Making } \\
\text { \& Planning }\end{array}$ & $\mathrm{N}$ & $\mathrm{N}$ & $\mathrm{N}$ & $\mathrm{N}$ & $\mathrm{N}$ & $\mathrm{Y}^{*}$ & $\mathrm{~N}$ & $\mathrm{Y}^{*}$ & $\mathrm{~N}$ & 2 \\
\hline 48. & $\begin{array}{l}\text { National } \\
\text { Libraries }\end{array}$ & $\mathrm{N}$ & $\mathrm{N}$ & $\mathrm{N}$ & $\mathrm{N}$ & $\mathrm{N}$ & $\mathrm{N}$ & $\mathrm{N}$ & $\mathrm{Y}^{*}$ & $\mathrm{~N}$ & 1 \\
\hline 49. & $\begin{array}{l}\text { Online } \\
\text { Information } \\
\text { Retrieval } \\
\end{array}$ & $\mathrm{N}$ & $\mathrm{N}$ & $\mathrm{N}$ & $\mathrm{N}$ & $\mathrm{N}$ & $\mathrm{Y}$ & $\mathrm{N}$ & & $\mathrm{N}$ & 1 \\
\hline 50. & $\begin{array}{l}\text { Personality Dev. } \\
\& \text { Comm. Skills }\end{array}$ & $\mathrm{N}$ & $\mathrm{N}$ & $\mathrm{N}$ & $\mathrm{N}$ & $\mathrm{N}$ & $\mathrm{N}$ & $\mathrm{N}$ & $\mathrm{Y}$ & $\mathrm{N}$ & 1 \\
\hline 51. & $\begin{array}{l}\text { Planning of Lib. } \\
\text { \& Info. Centers }\end{array}$ & $\mathrm{Y}$ & $\mathrm{N}$ & $\mathrm{N}$ & $\mathrm{N}$ & $\mathrm{N}$ & $\mathrm{N}$ & $\mathrm{N}$ & $\mathrm{N}$ & $\mathrm{N}$ & 1 \\
\hline 52. & Practicum & $\mathrm{N}$ & $\mathrm{Y}$ & $\mathrm{N}$ & $\mathrm{N}$ & $\mathrm{N}$ & $\mathrm{Y}$ & $\mathrm{N}$ & $\mathrm{N}$ & $\mathrm{N}$ & 2 \\
\hline 53. & $\begin{array}{l}\text { Preservation \& } \\
\text { Conservation }\end{array}$ & $\mathrm{N}$ & $\mathrm{N}$ & $\mathrm{Y}^{*}$ & $\mathrm{~N}$ & $\mathrm{Y}^{*}$ & $\mathrm{Y}^{*}$ & $\mathrm{~N}$ & $\mathrm{Y}^{*}$ & $\mathrm{~N}$ & 4 \\
\hline 54. & $\begin{array}{l}\text { Prof. } \\
\text { Communication } \\
\text { Skills }\end{array}$ & $\mathrm{N}$ & $\mathrm{Y}^{*}$ & $\mathrm{~N}$ & $\mathrm{~N}$ & $\mathrm{~N}$ & $\mathrm{~N}$ & $\mathrm{~N}$ & $\mathrm{~N}$ & $\mathrm{~N}$ & 1 \\
\hline 55. & $\begin{array}{l}\text { Project } \\
\text { Management }\end{array}$ & $\mathrm{N}$ & $\mathrm{Y}^{*}$ & $\mathrm{Y}^{*}$ & $\mathrm{~N}$ & $\mathrm{Y}^{*}$ & $\mathrm{Y}^{*}$ & $\mathrm{~N}$ & $\mathrm{Y}^{*}$ & $\mathrm{~N}$ & 5 \\
\hline 56. & $\begin{array}{l}\text { Public Libraries } \\
\& \text { Legislation }\end{array}$ & $\mathrm{Y}^{*}$ & $\mathrm{~N}$ & $\mathrm{~N}$ & $\mathrm{Y}^{*}$ & $\mathrm{~N}$ & $\mathrm{Y}^{*}$ & $\mathrm{~N}$ & $\mathrm{Y}^{*}$ & $\mathrm{~N}$ & 4 \\
\hline 57 & $\begin{array}{l}\text { Public Records, } \\
\text { Rare Materials }\end{array}$ & $\mathrm{N}$ & $\mathrm{N}$ & $\mathrm{N}$ & $\mathrm{N}$ & $\mathrm{N}$ & $\mathrm{Y}^{*}$ & Y2 & $\mathrm{Y}^{*}$ & $\mathrm{~N}$ & 4 \\
\hline 58. & $\begin{array}{l}\text { Qualitative Res. } \\
\text { Methods }\end{array}$ & $\mathrm{N}$ & $\mathrm{N}$ & $\mathrm{Y}$ & $\mathrm{N}$ & $\mathrm{Y}$ & $\mathrm{N}$ & $\mathrm{N}$ & $\mathrm{N}$ & $\mathrm{N}$ & 2 \\
\hline 59. & $\begin{array}{l}\text { Record } \\
\text { Management }\end{array}$ & $\mathrm{N}$ & $\mathrm{N}$ & $\mathrm{N}$ & $\mathrm{N}$ & $\mathrm{N}$ & $\mathrm{Y}^{*}$ & $\mathrm{~N}$ & $\mathrm{Y}^{*}$ & $\mathrm{~N}$ & 2 \\
\hline 60. & $\begin{array}{l}\text { Research } \\
\text { Methodology }\end{array}$ & $\mathrm{Y}$ & $\mathrm{N}$ & $\mathrm{N}$ & $\mathrm{Y}$ & $\mathrm{N}$ & $\mathrm{N}$ & Y2 & $\mathrm{N}$ & $\mathrm{N}$ & 4 \\
\hline 61. & $\begin{array}{l}\text { Research } \\
\text { Methods in LIS }\end{array}$ & $\mathrm{N}$ & $\mathrm{Y}$ & $\mathrm{N}$ & $\mathrm{N}$ & $\mathrm{N}$ & $\mathrm{Y}$ & $\mathrm{N}$ & $\mathrm{Y}$ & $\mathrm{N}$ & 3 \\
\hline 62. & Research Project & $\mathrm{N}$ & $\mathrm{N}$ & $\mathrm{Y}$ & $\mathrm{N}$ & $\mathrm{N}$ & $\mathrm{N}$ & $\mathrm{N}$ & $\mathrm{N}$ & $\mathrm{Y}^{*}$ & 2 \\
\hline 63. & $\begin{array}{l}\text { Resource } \\
\text { Sharing \& } \\
\text { Networking } \\
\end{array}$ & $\mathrm{Y}$ & $\mathrm{N}$ & $\mathrm{Y}$ & $\mathrm{Y}$ & $\mathrm{Y}$ & $\mathrm{Y}^{*}$ & $\mathrm{Y} 2$ & $\mathrm{Y}^{*}$ & $\mathrm{Y}$ & 9 \\
\hline 64. & $\begin{array}{l}\text { School Library } \\
\text { Media Center }\end{array}$ & $\mathrm{Y}^{*}$ & $\mathrm{Y}^{*}$ & $\mathrm{~N}$ & $\mathrm{~N}$ & $\mathrm{~N}$ & $\mathrm{Y}^{*}$ & $\mathrm{~N}$ & $\mathrm{Y}^{*}$ & $\mathrm{~N}$ & 4 \\
\hline 65. & Science \& & $\mathrm{N}$ & $\mathrm{N}$ & $\mathrm{N}$ & $\mathrm{N}$ & $\mathrm{N}$ & $\mathrm{Y}^{*}$ & $\mathrm{~N}$ & $\mathrm{Y}^{*}$ & $\mathrm{~N}$ & 2 \\
\hline
\end{tabular}




\begin{tabular}{|c|l|c|c|c|c|c|c|c|c|c|c|}
\hline & $\begin{array}{l}\text { Technology } \\
\text { Libraries }\end{array}$ & & & & & & & & & & \\
\hline 66. & $\begin{array}{l}\text { Semantic Web \& } \\
\text { Linked Data } \\
\text { Technologies }\end{array}$ & $\mathrm{N}$ & $\mathrm{Y}^{*}$ & $\mathrm{~N}$ & $\mathrm{~N}$ & $\mathrm{~N}$ & $\mathrm{~N}$ & $\mathrm{~N}$ & $\mathrm{~N}$ & $\mathrm{~N}$ & $\mathbf{1}$ \\
\hline 67. & $\begin{array}{l}\text { Serial } \\
\text { Management }\end{array}$ & $\mathrm{N}$ & $\mathrm{N}$ & $\mathrm{Y}^{*}$ & $\mathrm{~N}$ & $\mathrm{Y}^{*}$ & $\mathrm{Y}^{*}$ & $\mathrm{Y} 2$ & $\mathrm{Y}^{*}$ & $\mathrm{~N}$ & $\mathbf{6}$ \\
\hline 68. & Special Libraries & $\mathrm{Y}^{*}$ & $\mathrm{~N}$ & $\mathrm{~N}$ & $\mathrm{Y}^{*}$ & $\mathrm{~N}$ & $\mathrm{Y}^{*}$ & $\mathrm{~N}$ & $\mathrm{Y}^{*}$ & $\mathrm{~N}$ & $\mathbf{4}$ \\
\hline 69. & $\begin{array}{l}\text { System Analysis } \\
\text { \& Design }\end{array}$ & $\mathrm{N}$ & $\mathrm{N}$ & $\mathrm{N}$ & $\mathrm{N}$ & $\mathrm{N}$ & $\mathrm{Y}^{*}$ & $\mathrm{~N}$ & $\mathrm{Y}^{*}$ & $\mathrm{~N}$ & $\mathbf{2}$ \\
\hline 70. & Thesis & $\mathrm{Y}^{*}$ & $\mathrm{Y}^{*}$ & $\mathrm{~N}$ & $\mathrm{Y}^{*}$ & $\mathrm{Y}$ & $\mathrm{Y}^{*}$ & $\mathrm{Y}^{*}$ & $\mathrm{Y}^{*}$ & $\mathrm{~N}$ & $\mathbf{7}$ \\
\hline 71. & $\begin{array}{l}\text { Web } \\
\text { Development }\end{array}$ & $\mathrm{N}$ & $\mathrm{N}$ & $\mathrm{Y}^{*}$ & $\mathrm{~N}$ & $\mathrm{Y}^{*}$ & $\mathrm{~N}$ & $\mathrm{~N}$ & $\mathrm{~N}$ & $\mathrm{~N}$ & $\mathbf{2}$ \\
\hline 72. & Web Publishing & $\mathrm{N}$ & $\mathrm{N}$ & $\mathrm{N}$ & $\mathrm{N}$ & $\mathrm{N}$ & $\mathrm{Y}^{*}$ & $\mathrm{~N}$ & $\mathrm{Y}^{*}$ & $\mathrm{~N}$ & $\mathbf{2}$ \\
\hline & Total & $\mathbf{1 9}$ & $\mathbf{2 0}$ & $\mathbf{2 1}$ & $\mathbf{1 4}$ & $\mathbf{2 2}$ & $\mathbf{4 3}$ & $\mathbf{1 1}$ & $\mathbf{4 1}$ & $\mathbf{1 3}$ & $\mathbf{2 0 4}$ \\
\hline
\end{tabular}

$\mathrm{Y}^{*}=$ optional course $\quad * *$ Science, Technology, Social Science, Humanities, Islam, Pakistan ^ Information Management Y2 = Two courses under same title as I \& II

The above 72 titles of courses (in all) are being offered at M.A (Final) or equivalent level by the nine library schools of Pakistan. Some of the courses are common and are taught in majority of the library schools but most of the courses are taught independently or in two to three library schools. The majority of the courses of final year are optional or elective; hence the number of the total courses rises in each library school. Allama Iqbal Open University (AIOU), Islamabad offers five pairs of courses under same title but in fact, they are ten courses. Hence these courses are counted as two courses each against the same title. Archives \& Record Management, Resource Sharing and Networking, and Information Sources and Literature are the courses being offered by eight out of nine library schools. Advanced Technical Operations is being offered by five library schools (either as compulsory or elective course) except Karachi University, Sindh University, Baluchistan University, and Khushal Khan Khattak University. Sindh University, Baluchistan University, and KKK University are not offering this course at any level whereas Karachi University offers the course as two different courses i.e. "Comparative Classification" and "Cataloguing of Special Materials". The courses of Information Sources and Literatures on Islam, Pakistan, Humanities, Social Science, Science, and Technology are also offered by all the universities (as elective courses) except Punjab University and AIOU. AIOU does not offer these optional courses whereas Punjab University offers these courses under different titles like Business and Industry Information, Government Information, Health Sciences Information, Humanities and Social Sciences Information, Law Information, and Scientific and Technical Information. All universities, except Punjab University, are offering the course of Resource Sharing and Networking whereas AIOU offers this course into two parts i.e. I and II. M.A. 
thesis as an elective course is practiced by all the library schools under the same title whereas Sindh University, and KKK University offer it under the title Research Project.

\section{Modern especially ICT-related Courses offered in the Curricula}

The analysis of the courses taught at the departments of Library \& Information Science/ Information Management of Pakistani universities, shows the following ICT-related courses in the curricula.

Table-6 Modern Components/Trends at Postgraduate Level in LIS Curricula in Pakistan

\begin{tabular}{|c|c|c|c|c|c|c|c|c|c|}
\hline Trends & KU & PuU & SiU & PeU & BU & IU & AU & SaU & KK \\
\hline Digital Library & $\checkmark$ & $\checkmark$ & $\checkmark$ & $\mathrm{x}$ & $\mathrm{x}$ & $\checkmark$ & $\mathrm{x}$ & $\checkmark$ & $\checkmark$ \\
\hline $\begin{array}{l}\text { E-Learning/E-Collection } \\
\text { Mngmt. }\end{array}$ & $x$ & $\checkmark$ & $x$ & $x$ & $\mathrm{x}$ & $\checkmark$ & $\mathrm{x}$ & $x$ & $x$ \\
\hline $\begin{array}{l}\text { Web } \\
\text { Designing/Publishing }\end{array}$ & $x$ & $\checkmark$ & $x$ & $x$ & $\mathrm{x}$ & $\checkmark$ & $\mathrm{x}$ & $\mathrm{x}$ & $x$ \\
\hline Information Architecture & $\mathrm{x}$ & $\sqrt{ }$ & $\mathrm{x}$ & $\mathrm{x}$ & $\mathrm{X}$ & $\checkmark$ & $\mathrm{X}$ & $\mathrm{x}$ & $\mathrm{X}$ \\
\hline Information Retrieval & $\checkmark$ & $\checkmark$ & $\checkmark$ & $\mathrm{x}$ & $\mathrm{x}$ & $\checkmark$ & $\mathrm{x}$ & $\mathrm{x}$ & $\mathrm{x}$ \\
\hline Database Designing & $\checkmark$ & $\mathrm{x}$ & $\mathrm{x}$ & $\mathrm{x}$ & $\mathrm{x}$ & $\checkmark$ & $\mathrm{x}$ & $\mathrm{x}$ & $\mathrm{x}$ \\
\hline $\begin{array}{l}\text { ICT/Introduction to } \\
\text { Computers }\end{array}$ & $\checkmark$ & $\checkmark$ & $\checkmark$ & $\sqrt{ }$ & $\checkmark$ & $\checkmark$ & $\checkmark$ & $\checkmark$ & $\checkmark$ \\
\hline
\end{tabular}

Key:

KU = Karachi University, Karachi

$\mathrm{PuU}=$ Punjab University, Lahore

$\mathrm{SiU}=$ Sindh University, Jamshoro

$\mathrm{PeU}=$ Peshawar University, Peshawar

$\mathrm{BU}=$ Baluchistan University, Questta

IU = Islamia University, Bahawalpur

$\mathrm{AU}=$ Allama Iqbal Open University, Isl

$\mathrm{SaU}$ = Sargodha University, Sargodha

KK = Khushal Khan Khattak University, Karak

\section{Elective (Optional) Courses}

At postgraduate (Masters) level, the students are given choice to opt one or some courses of their interest or aptitude out of given list of courses. In library and information science, this facility is available by all the universities. The Pakistani universities are offering compulsory as well as optional courses to their students. Following is a detail about elective courses: 
Jhss, Vol. 9, No. 1, January to June, 2018

Table-7 Number of optional courses offered in LIS schools

\begin{tabular}{|c|c|c|c|c|}
\hline \multirow[t]{2}{*}{ University } & \multicolumn{3}{|c|}{ Number of Elective Courses Taught } & \multirow{2}{*}{$\begin{array}{l}\text { Number } \\
\text { of } \\
\text { Choices } \\
\text { given for } \\
\text { Elective } \\
\text { Courses } \\
\end{array}$} \\
\hline & $\begin{array}{l}3^{\text {rdSemester/ }} \\
7^{\text {th }} \text { Semester }\end{array}$ & $\begin{array}{l}4^{\text {th }} \text { Semester/ } \\
8^{\text {th }} \text { Semester }\end{array}$ & Annual & \\
\hline $\begin{array}{l}\text { Karachi } \\
\text { University, } \\
\text { Karachi }\end{array}$ & $1(3)+1(3)$ & $1(3)+1(4)+1(2)$ & & $5(15)$ \\
\hline $\begin{array}{l}\text { Punjab } \\
\text { University, } \\
\text { Lahore }\end{array}$ & Nil & $6(13)$ & & $6(13)$ \\
\hline $\begin{array}{l}\text { Sindh } \\
\text { University, } \\
\text { Jamshoro }\end{array}$ & $2(7)$ & $2(7)$ & & $4(14)$ \\
\hline $\begin{array}{l}\text { Peshawar } \\
\text { University } \\
\text { Peshawar }\end{array}$ & & & $\begin{array}{l}1(3)+ \\
1(8)\end{array}$ & $2(11)$ \\
\hline $\begin{array}{l}\text { Baluchistan } \\
\text { University } \\
\text { Quetta }\end{array}$ & $2(20)$ & $2(20)$ & & $4(20)$ \\
\hline $\begin{array}{l}\text { Islamia } \\
\text { University } \\
\text { Bahawalpur } \\
\end{array}$ & $2(5)$ & $1(10)+1(7)+1(7)+2(14)$ & & $7(43)$ \\
\hline $\begin{array}{l}\text { AIOU, } \\
\text { Islamabad }\end{array}$ & Nil & $4(5)$ or $1(5)$ & & $4(5) / 1(5)$ \\
\hline $\begin{array}{l}\text { Sargodha } \\
\text { University, } \\
\text { Sargodha } \\
\end{array}$ & $2(6)$ & $1(10)+1(7)+1(6)+2(13)$ & & $7(42)$ \\
\hline $\begin{array}{l}\text { Khushal } \\
\text { Khattak Univ. } \\
\text { Karak }\end{array}$ & $1(5)$ & $1(2)$ & & $2(7)$ \\
\hline
\end{tabular}

\section{Titles of Elective Courses}

All the universities of Pakistan are offering more or less similar titles of courses as optional courses but the choices may slightly vary depending upon the local market needs. In urban areas, as the libraries associated with medical, business and management, law, banks and financial institutions, and other special organizations are well developed. In industrial areas, the scientific and technical libraries, and in 
rural areas, agricultural libraries are the need of the people. In federal and provincial capital cities, the libraries of parliament and assemblies are also established whereas public, school, children, and academic libraries are the need of all the areas so the library schools covering the libraries of their jurisdiction, offer optional courses of librarianship and information sources as per their need. Following elective courses are being offered by the Pakistani library schools:

Table-8 Titles of elective courses offered by the library schools

\begin{tabular}{|l|l|}
\hline Name & of \\
\hline Karachitles University & $\begin{array}{l}\text { Any One from the following: } \\
\text { Information Sources \& Literature on Humanities } \\
\text { Information Sources \& Literature on Science } \\
\text { Information Sources \& Literature on Islam }\end{array}$ \\
& $\begin{array}{l}\text { Any One from the following: } \\
\text { Public Library, Library Legislation and System } \\
\text { Children Librarianship, School Library Media Center } \\
\text { Special Librarianship }\end{array}$ \\
& $\begin{array}{l}\text { Any One from the following: } \\
\text { Information Sources \& Literature on Social Science } \\
\text { Information Sources \& Literature on Technology } \\
\text { Information Sources \& Literature on Pakistan }\end{array}$ \\
& $\begin{array}{l}\text { Any One from the following: } \\
\text { Archive Management and Preservation } \\
\text { Academic Libraries } \\
\text { Medical Libraries and Librarianship }\end{array}$ \\
\hline Punjab University & $\begin{array}{l}\text { Any Six from the following: } \\
\text { Digital Libraries } \\
\text { Project Management } \\
\text { Advanced Cataloguing and Classification } \\
\text { Indexing and Abstracting } \\
\text { Scientific and Technical Information Sources } \\
\text { Electronic Resources Management } \\
\text { Advance Management \& Leadership Skills } \\
\text { Application of Information Systems } \\
\text { Semantic Web and Linked Data Technologies } \\
\text { Professional Communication Skills } \\
\text { School Library Media Center } \\
\text { Media Information Management }\end{array}$ \\
\hline
\end{tabular}


Jhss, Vol. 9, No. 1 , January to June, 2018

\begin{tabular}{|c|c|}
\hline & Thesis (6 credits) \\
\hline Sindh University & $\begin{array}{l}\text { Any Two from the following: } \\
\text { Bibliographical Control } \\
\text { Digital Library } \\
\text { Inf. Sources on Science, Technology, Humanities, Islam, } \\
\text { Pakistan } \\
\text { Intellectual Property Rights } \\
\text { Project Management } \\
\text { Serial Management } \\
\text { Database Design and Management } \\
\text { Any Two from the following: } \\
\text { Web Development } \\
\text { Knowledge Management } \\
\text { Human Resource Management } \\
\text { Financial Management } \\
\text { Archives and Records Management } \\
\text { Preservation and Conservation of Library Material } \\
\text { Media Librarianship }\end{array}$ \\
\hline $\begin{array}{l}\text { Peshawar } \\
\text { University }\end{array}$ & $\begin{array}{l}\text { Any One from the following: } \\
\text { Information Sources of Science and Technology } \\
\text { Information Sources of Humanities and Social Sciences } \\
\text { Information Sources of Islam and Pakistan } \\
\text { Any One from the following: } \\
\text { Public Records, Rare and Archival Materials and } \\
\text { Preservation } \\
\text { Communication and Media Librarianship } \\
\text { Management of Information Systems } \\
\text { Special Libraries } \\
\text { Academic Libraries } \\
\text { Public Libraries and Legislation } \\
\text { Marketing of Library Information Services } \\
\text { Thesis }\end{array}$ \\
\hline $\begin{array}{l}\text { Baluchistan } \\
\text { University }\end{array}$ & $\begin{array}{l}\text { Any Four from the following: } \\
\text { Digital Libraries } \\
\text { Media Librarianship } \\
\text { Data Base Design \& Management } \\
\text { Web Development } \\
\text { Knowledge Management } \\
\text { Human Resource Management } \\
\text { Financial Management }\end{array}$ \\
\hline
\end{tabular}




\begin{tabular}{|c|c|}
\hline & $\begin{array}{l}\text { Archives \& Records Management } \\
\text { Intellectual Property Rights } \\
\text { Bibliographic Control } \\
\text { Project Management } \\
\text { Preservation \& Conservation of Library Material } \\
\text { Serial Management } \\
\text { Information Sources on Islam and Pakistan } \\
\text { Information Sources on Business \& Industry } \\
\text { Information Sources on Health Sciences } \\
\text { Information Sources on Humanities and Social Sciences } \\
\text { Information Sources on Science \& Technology } \\
\text { Information Sources on Law } \\
\text { Information Sources on Agriculture }\end{array}$ \\
\hline Islamia University & $\begin{array}{l}\text { Any Two from the following: } \\
\text { Literature on Humanities } \\
\text { Literature on Social Sciences } \\
\text { Literature on Science } \\
\text { Literature on Technology } \\
\text { Literature on Pakistan } \\
\text { Any One from the following: } \\
\text { Academic Libraries } \\
\text { School Libraries } \\
\text { Children Libraries } \\
\text { Public Libraries } \\
\text { Special Libraries } \\
\text { Agricultural Libraries } \\
\text { Health and Medical Libraries } \\
\text { Law Libraries } \\
\text { Science and Technology Libraries } \\
\text { Any One from the following: } \\
\text { Advanced Management Techniques } \\
\text { Public Record, Rare Materials and their Conservation } \\
\text { Communication and Media Librarianship } \\
\text { Indexing and Abstracting } \\
\text { Resource Sharing and Networking } \\
\text { International and Comparative Librarianship } \\
\text { Models for Library Management, Decision Making and } \\
\text { Planning } \\
\text { Any One from the following: } \\
\text { Web Publishing } \\
\text { Database Structure and design }\end{array}$ \\
\hline
\end{tabular}




\begin{tabular}{|c|c|}
\hline & $\begin{array}{l}\text { Computer Networks } \\
\text { Computer Programming for Information Management } \\
\text { System Analysis and Design } \\
\text { Digital Libraries } \\
\text { Electronic Collection Management } \\
\text { Any Two from the following: } \\
\text { Serials Management } \\
\text { Information Policy } \\
\text { Knowledge Management } \\
\text { Financial Management Libraries and Information Centers } \\
\text { Information Seeking and Use } \\
\text { Project Management } \\
\text { Archives Management } \\
\text { Advanced Cataloguing and Classification } \\
\text { Information Architecture } \\
\text { Information Literacy Instruction } \\
\text { Records Management } \\
\text { Preservation and Conservation of Library and Archival } \\
\text { Material } \\
\text { Human Resource Management } \\
\text { Thesis and Viva Voce }\end{array}$ \\
\hline AIOU & $\begin{array}{l}\text { No Elective Courses except in lieu of following four } \\
\text { courses of last semester, the students may opt thesis } \\
\text { comprising } 12 \text { credit hours: } \\
\text { Public Records, Rare Material \& their Conservation-I } \\
\text { Public Records, Rare Material \& their Conservation-II } \\
\text { Management of Serials Publications-I } \\
\text { Management of Serials Publications-II }\end{array}$ \\
\hline $\begin{array}{l}\text { Sargodha } \\
\text { University }\end{array}$ & $\begin{array}{l}\text { Any Two from the following: } \\
\text { Literature on Islam } \\
\text { Literature on Pakistan } \\
\text { Literature on Humanities } \\
\text { Literature on Social Sciences } \\
\text { Literature on Science } \\
\text { Literature on Technology } \\
\text { Any One from the following: } \\
\text { National Libraries } \\
\text { Academic Libraries } \\
\text { School Libraries }\end{array}$ \\
\hline
\end{tabular}




\begin{tabular}{|c|c|}
\hline & $\begin{array}{l}\text { Children Libraries } \\
\text { Public Libraries } \\
\text { Special Libraries } \\
\text { Agricultural Libraries } \\
\text { Health and Medical Libraries } \\
\text { Law Libraries } \\
\text { Science and Technology Libraries } \\
\text { Any One from the following: } \\
\text { Advanced Management Techniques } \\
\text { Public Records, Rare Materials and their Conservation } \\
\text { Communication and Media Librarianship } \\
\text { Indexing and Abstracting } \\
\text { Libraries and Society } \\
\text { International and Comparative Librarianship } \\
\text { Models for Library Management, Decision Making and } \\
\text { Planning } \\
\text { Any One from the following: } \\
\text { Web Publishing } \\
\text { Database Structure and Design } \\
\text { Computer Networks } \\
\text { Computer Programming for Information Management } \\
\text { Systems Analysis and Design } \\
\text { Digital Libraries } \\
\text { Any Two from the following: } \\
\text { Information Policy } \\
\text { Knowledge Management } \\
\text { Financial Management of Libraries and Information Centers } \\
\text { Information Seeking and Use } \\
\text { Project Management } \\
\text { Archives Management } \\
\text { Advanced Cataloguing and Classification } \\
\text { Resource Sharing and Networking } \\
\text { Records Management } \\
\text { Serials Management } \\
\text { Preservation and Conservation of Library and Archival } \\
\text { Material } \\
\text { Human Resource Management } \\
\text { Thesis and Viva Voce (6 credits) }\end{array}$ \\
\hline $\begin{array}{l}\text { Khushal Khan } \\
\text { Khattak } \\
\text { Karak }\end{array}$ & $\begin{array}{l}\text { Any One from the following: } \\
\text { Information Sources and Literature on Social Science } \\
\text { Information Sources and Literature on Islam and Pakistan }\end{array}$ \\
\hline
\end{tabular}




\begin{tabular}{|l|l|}
\hline & $\begin{array}{l}\text { Information Sources and Literature on Science and } \\
\text { Technology } \\
\text { Information Sources and Literature on Law } \\
\text { Bibliographical Control }\end{array}$ \\
$\begin{array}{l}\text { In lieu of Research Project, following Two courses are } \\
\text { to be opted } \\
\text { Library and Information Software Packages (Theory \& } \\
\text { Practical) } \\
\text { Information Seeking Behavior }\end{array}$ \\
\hline
\end{tabular}

\section{Practical Component in Curricula}

The LIS curriculum is composed of both theoretical and practical components. Nature of some of the courses is fully practical-based whereas that of some other courses is theory-cum-practical.

Table-9 Theory-Practical Components of the Courses

\begin{tabular}{|c|c|c|c|c|c|c|c|c|c|c|c|c|c|c|c|}
\hline \multirow[t]{2}{*}{ Uni } & \multicolumn{3}{|c|}{$1^{\text {st }}$ Semester } & \multicolumn{3}{|c|}{$\begin{array}{l}2^{\text {nd }} \\
\text { Semester }\end{array}$} & \multicolumn{3}{|c|}{$\begin{array}{l}3^{\text {rd }} \\
\text { Semester }\end{array}$} & \multicolumn{3}{|c|}{$\begin{array}{l}4^{\text {th }} \\
\text { Semester }\end{array}$} & \multicolumn{3}{|c|}{ Annual } \\
\hline & $\begin{array}{l}\text { T } \\
\mathbf{h}\end{array}$ & Pr & $\begin{array}{l}\mathrm{T} / \\
\mathrm{P}\end{array}$ & $\begin{array}{l}\mathbf{T} \\
\mathbf{h}\end{array}$ & $\begin{array}{l}\mathbf{P} \\
\mathbf{r}\end{array}$ & $\begin{array}{l}\mathrm{T} / \\
\mathrm{P}\end{array}$ & $\begin{array}{l}\mathrm{T} \\
\mathrm{h}\end{array}$ & $\begin{array}{l}\mathbf{P} \\
\mathbf{r}\end{array}$ & $\begin{array}{l}\mathrm{T} / \\
\mathrm{P}\end{array}$ & $\begin{array}{l}\mathbf{T} \\
\mathbf{h}\end{array}$ & $\begin{array}{l}\mathbf{P} \\
\mathbf{r}\end{array}$ & $\begin{array}{l}\mathrm{T} / \\
\mathrm{P}\end{array}$ & Th & Pr & $\begin{array}{l}\mathrm{T} / \\
\mathrm{P}\end{array}$ \\
\hline $\mathrm{KU}$ & 6 & - & - & 3 & 2 & 2 & 5 & - & 2 & 4 & - & 3 & $\mathrm{x}$ & $\mathrm{x}$ & $\mathrm{x}$ \\
\hline $\begin{array}{l}\mathrm{Pu} \\
\mathrm{U}\end{array}$ & 5 & - & 2 & 3 & 2 & 1 & 5 & - & 1 & 7 & 3 & 3 & $\mathrm{x}$ & $\mathrm{X}$ & $\mathrm{x}$ \\
\hline $\mathrm{SiU}$ & 4 & 1 & - & 3 & 1 & 1 & 3 & - & 2 & - & 1 & 4 & $\mathrm{x}$ & $\mathrm{X}$ & $\mathrm{x}$ \\
\hline $\begin{array}{l}\mathrm{Pe} \\
\mathrm{U} \\
\end{array}$ & $\mathrm{x}$ & $\mathrm{x}$ & $\mathrm{x}$ & $\mathrm{x}$ & $\bar{x}$ & $\mathrm{x}$ & $\mathrm{x}$ & $\mathrm{X}$ & $\mathrm{x}$ & $\mathrm{x}$ & $\mathrm{x}$ & $\mathrm{x}$ & $\begin{array}{c}5+ \\
4 \\
\end{array}$ & $\begin{array}{c}0+ \\
4 \\
\end{array}$ & $\begin{array}{c}1+ \\
1 \\
\end{array}$ \\
\hline $\mathrm{BU}$ & 4 & 1 & - & 3 & 1 & 1 & 5 & - & 1 & 2 & - & 2 & $\mathrm{x}$ & $\mathrm{x}$ & $\mathrm{x}$ \\
\hline IU & 4 & - & 2 & 2 & 2 & 2 & 4 & - & 1 & 5 & 1 & - & $\mathrm{x}$ & $\mathrm{x}$ & $\mathrm{x}$ \\
\hline $\mathrm{AU}$ & 3 & - & 2 & 3 & - & 2 & 2 & 2 & 2 & 4 & - & 1 & $\mathrm{x}$ & $\mathrm{x}$ & $\mathrm{x}$ \\
\hline $\begin{array}{l}\text { Sa } \\
\mathrm{U}\end{array}$ & 4 & - & 2 & 3 & 2 & 1 & 5 & - & 1 & 5 & 1 & - & $\mathrm{x}$ & $\mathrm{x}$ & $\mathrm{x}$ \\
\hline KK & 6 & - & - & 2 & 2 & 2 & 4 & - & 2 & 3 & 1 & 2 & $\mathrm{x}$ & $\mathrm{x}$ & $\mathrm{x}$ \\
\hline
\end{tabular}

"Th" indicates Theory

"Pr" indicates Practical

"T/P" indicates Theory-cum-Practical

Key:

$\mathrm{KU}=$ Karachi University, Karachi

$\mathrm{PuU}=$ Punjab University, Lahore 
$\mathrm{SiU}=$ Sindh University, Jamshore

$\mathrm{PeU}=$ Peshawar University, Peshawar

$\mathrm{BU}=$ Baluchistan University, Questta

IU = Islamia University, Bahawalpur

$\mathrm{AU}=$ Allama Iqbal Open University, Isl

$\mathrm{SaU}=$ Sargodha University, Sargodha

KK = Khushal Khan Khattak University, Karak

\section{Teaching Methods}

Both traditional and modern teaching methods are applied in all the universities of Pakistan. Nature of some courses demands the conventional style of teaching in which the focus is on teachers' lecturing but the interaction and participation of the pupils is also required. Some other courses demand the mediocre and modern styles of teaching.

\section{Table-10 Teaching Methods}

\begin{tabular}{|c|c|c|c|c|c|c|c|c|c|}
\hline Teaching Methods & $\mathbf{K U}$ & PuU & SiU & PeU & BU & IU & AU & SaU & KK \\
\hline Lectures & $\checkmark$ & $\checkmark$ & $\checkmark$ & $\checkmark$ & $\checkmark$ & $\checkmark$ & $\checkmark$ & $\checkmark$ & $\checkmark$ \\
\hline Lectures-cum-Discussion & $\checkmark$ & $\checkmark$ & $\checkmark$ & $\checkmark$ & $\checkmark$ & $\checkmark$ & $\checkmark$ & $\checkmark$ & $\checkmark$ \\
\hline Group Discussion & $\checkmark$ & $\checkmark$ & $\checkmark$ & $\checkmark$ & $\checkmark$ & $\checkmark$ & $\checkmark$ & $\checkmark$ & $\checkmark$ \\
\hline Tutorials & $\mathrm{x}$ & $\mathrm{x}$ & $\mathrm{x}$ & $\checkmark$ & $\checkmark$ & $\mathrm{x}$ & $\checkmark$ & $\mathrm{x}$ & $\mathrm{x}$ \\
\hline Case Study & $\mathrm{x}$ & $\mathrm{x}$ & $\mathrm{x}$ & $\checkmark$ & $\checkmark$ & $\mathrm{x}$ & $\checkmark$ & $\mathrm{x}$ & $\mathrm{x}$ \\
\hline Assignments & $\checkmark$ & $\checkmark$ & $\checkmark$ & $\checkmark$ & $\checkmark$ & $\checkmark$ & $\checkmark$ & $\checkmark$ & $\checkmark$ \\
\hline Seminars & 0 & 0 & $\mathrm{x}$ & $\checkmark$ & $\checkmark$ & 0 & $\checkmark$ & $\checkmark$ & $\checkmark$ \\
\hline Panels of Experts & 0 & 0 & $\mathrm{x}$ & $\checkmark$ & $\checkmark$ & 0 & $\checkmark$ & $\mathrm{x}$ & $\mathrm{x}$ \\
\hline Brainstorming & $\mathrm{x}$ & $\mathrm{x}$ & $\mathrm{x}$ & $\mathrm{x}$ & $\mathrm{x}$ & $\mathrm{x}$ & $\mathrm{x}$ & $\mathrm{x}$ & $\mathrm{x}$ \\
\hline Group Work & $\checkmark$ & $\checkmark$ & $\checkmark$ & $\mathrm{x}$ & $\mathrm{x}$ & $\checkmark$ & $\mathrm{x}$ & $\mathrm{x}$ & $\checkmark$ \\
\hline Online Discussion & $\mathrm{x}$ & $\mathrm{x}$ & $\mathrm{x}$ & $\mathrm{x}$ & $\mathrm{x}$ & $\mathrm{x}$ & $\mathrm{x}$ & $\mathrm{x}$ & $\mathrm{x}$ \\
\hline Guest Speakers & 0 & $\checkmark$ & $\mathrm{x}$ & $\checkmark$ & $\mathrm{x}$ & $\checkmark$ & $\mathrm{x}$ & $\checkmark$ & $\mathrm{x}$ \\
\hline Lab Exercises & $\checkmark$ & $\checkmark$ & $\checkmark$ & $\checkmark$ & $\checkmark$ & $\checkmark$ & $\checkmark$ & $\checkmark$ & $\checkmark$ \\
\hline Study Tours & 0 & 0 & 0 & $\checkmark$ & 0 & 0 & 0 & $\checkmark$ & $\mathrm{x}$ \\
\hline Presentations & $\checkmark$ & $\checkmark$ & $\checkmark$ & $\checkmark$ & $\checkmark$ & $\checkmark$ & $\checkmark$ & $\checkmark$ & $\checkmark$ \\
\hline
\end{tabular}

$$
\checkmark=\text { Yes } \quad \mathrm{x}=\text { No o }=\text { Occasionally }
$$

\section{Students Evaluation/Assessment Methods}

System of examinations in all the universities is more or less same i.e. Theory and Practical examinations are taken separately. But few other methods or systems are 
Jhss, Vol. 9, No. 1, January to June, 2018

also adopted in different courses. The following table defines clearly the assessment methods in every library school:

Table-11 System and Methods of Assessment

\begin{tabular}{|l|c|c|c|c|c|c|c|c|c|}
\hline $\begin{array}{l}\text { Evaluation/Assessment } \\
\text { Methods }\end{array}$ & KU & PuU & SiU & PeU & BU & IU & AU & SaU & KK \\
\hline Written Examinations & Y & Y & Y & Y & Y & Y & Y & Y & Y \\
\hline Case Study & N & N & N & N & N & N & N & N & N \\
\hline Oral Presentations & N & N & N & N & N & N & Y & Y & Y \\
\hline Formal Reports & O & O & N & N & N & Y & Y & Y & N \\
\hline Individual Works & O & O & N & N & N & Y & Y & N & N \\
\hline Group Projects & O & O & N & N & N & N & N & N & O \\
\hline Practical Works & Y & Y & Y & Y & Y & Y & Y & Y & Y \\
\hline Thesis and Viva Voce & O & O & O & O & O & O & Y & Y & Y \\
\hline
\end{tabular}

$$
\mathrm{Y}=\text { Yes } \quad \mathrm{X}=\mathrm{NoO}=\text { Occasionally }
$$

\section{Teaching Facilities/Aids}

All the universities are trying their best to provide better teaching facilities and well-equipped libraries and laboratories to their students.

Table-12 Teaching Facilities

\begin{tabular}{|c|c|c|c|c|c|c|c|c|c|}
\hline Teaching Facilities/Aids & $\mathbf{K U}$ & PuU & SiU & PeU & BU & IU & AU & SaU & KK \\
\hline Black/White Board & $\checkmark$ & $\checkmark$ & $\checkmark$ & $\checkmark$ & $\checkmark$ & $\checkmark$ & $\checkmark$ & $\checkmark$ & $\checkmark$ \\
\hline Overhead Projectors & $\checkmark$ & $\checkmark$ & $\checkmark$ & $\mathrm{x}$ & $\mathrm{x}$ & $\mathrm{x}$ & $\checkmark$ & $\mathrm{x}$ & $\mathrm{x}$ \\
\hline LCDs & $\mathrm{x}$ & $\mathrm{x}$ & $\mathrm{x}$ & $\mathrm{x}$ & $\mathrm{x}$ & $\mathrm{x}$ & $\checkmark$ & $\checkmark$ & $\mathrm{x}$ \\
\hline Multimedia Presentation & $\checkmark$ & $\checkmark$ & $\checkmark$ & $\checkmark$ & $\checkmark$ & $\checkmark$ & $\checkmark$ & $\checkmark$ & $\checkmark$ \\
\hline $\begin{array}{l}\text { Cataloguing/Classification } \\
\text { Lab }\end{array}$ & $\checkmark$ & $\checkmark$ & $\mathrm{x}$ & $\mathrm{x}$ & $\mathrm{x}$ & $\mathrm{x}$ & $\mathrm{x}$ & $\checkmark$ & $\mathrm{x}$ \\
\hline $\begin{array}{l}\text { Cataloguing/Classification } \\
\text { Tools }\end{array}$ & $\checkmark$ & $\checkmark$ & $\checkmark$ & $\checkmark$ & $\checkmark$ & $\checkmark$ & $\checkmark$ & $\checkmark$ & $\checkmark$ \\
\hline Computer Lab & $\checkmark$ & $\checkmark$ & $\checkmark$ & $\checkmark$ & $\checkmark$ & $\checkmark$ & $\mathrm{x}$ & $\checkmark$ & $\mathrm{x}$ \\
\hline $\begin{array}{l}\text { Departmental/Seminar } \\
\text { Library }\end{array}$ & $\checkmark$ & $\checkmark$ & $\checkmark$ & $\checkmark$ & $\checkmark$ & $\mathrm{x}$ & $\mathrm{x}$ & $\mathrm{x}$ & $\mathrm{x}$ \\
\hline $\begin{array}{l}\text { Well-equipped Audiovisual } \\
\text { Lab }\end{array}$ & $\mathrm{x}$ & $\mathrm{x}$ & $\mathrm{x}$ & $\checkmark$ & $\mathrm{x}$ & $\mathrm{x}$ & $\mathrm{x}$ & $\checkmark$ & $\mathrm{x}$ \\
\hline Sound System & $\checkmark$ & $\checkmark$ & $\checkmark$ & $\checkmark$ & $\mathrm{x}$ & $\mathrm{x}$ & $\checkmark$ & $\checkmark$ & $x$ \\
\hline
\end{tabular}


Both modern and traditional teaching facilities/aids are available in all the universities. Black/white board, the basic necessity of any educational institution, is used by all library schools. As overhead projector has been replaced by multimedia, so only three library schools i.e. Karachi, Punjab, and Sindh are still using it as well as multimedia which is under use of all library schools. Only two library schools i.e. Peshawar and Sargodha are maintaining well-equipped audiovisual lab where besides other equipment, LCD is also available. All schools are imparting training to their students with latest editions of all cataloguing and classification tools. Only three departments are having the facilities of separate cataloguing/classification labs whereas remaining departments are providing these services to their students either in the classrooms or in the departmental or central library. Computer labs are also maintained by all library schools except Allama Iqbal Open University.

The library education in Pakistan is heading towards right direction and not only public sector but also the private sector universities are taking interest in opening library and information science departments. Of nine public sector universities, only one university is offering only 4-year BS program and only one university is offering one-year each BLIS and MLIS programs. Four universities are offering 2-year Masters program, and three universities are practicing both 2-year and 4-year programs. As far as examination system is concerned, only Peshawar University is following annual system whereas remaining eight universities are practicing semester system. Number of courses offered in each semester is more or less equal in all the universities. The core and technical courses are taught by all the universities whereas specialized and elective courses are somewhat different but majority of the courses are same. Most of the elective courses meet the local needs of the library schools. All the library schools are focused on offering more and more ICT-related courses to meet the national and international demands. In designing and developing curriculum, the library schools pay special attention towards practical component of the courses. All library schools are endeavoring their best to adopt modern teaching methods and facilities. Students' performance is evaluated using all possible assessment methods. In short, the library education and library schools of Pakistan are on the right track and meeting the challenges of international market. To maintain the international repute, regular revision and updating of curricula, training facilities to faculty members, use of modern technology, and promotion of research activities are necessary. 
Jhss, Vol. 9, No. 1 , January to June, 2018

\section{Conclusion}

The education of library science (now library and information science) was started in 1956 from University of Karachi. At that time it was a one-year postgraduate program and gradually it was elevated to higher level and today has reached to Ph.D. Till 1982, five universities were offering programs in library science but later on, the number started increasing. The other universities of Pakistan, following the example of Karachi University, also started the programs and now nine public sector and three private sector universities of Pakistan are offering professional degree program in library and information science. Realizing the importance of library education, the private universities have started offering the program up to Ph.D.

Although the program had been started as one year postgraduate diploma in Library Science (PGD-LS), or DLS, or BLS, or equivalent but after some time, it had been converted into one more year as MLS, or MA in Library Science but in the recent years, due to the involvement of Higher Education Commission (HEC), emphasis was given to 4-year BS program (equivalent to M.A.). Some universities adopted the four-year program whereas some others continued with the two-year postgraduate program. Today, a few universities are practicing 2-year, some other 4-year, and some both the systems in parallel. As far as the system of education and examination is concerned, till the end of 2016, except two universities (Peshawar University and Baluchistan University) were practicing semester system whereas these universities were following annual system but with the start of 2017, Baluchistan University has also come in the mainstream and has adopted semester system and now only Peshawar University is practicing annual system.

The graduates of the universities who had/have two degrees of one year each (most commonly BLIS and MLIS) are in favor of continuation of these two separate programs as after successful completion of one year, if the student wants to drop for a reason or another, he/she can get a degree which would be useful for his/her career. Most of the working librarians support this practice and demand all the universities to follow the system.

As far as accreditation body to accredit a professional degree program is concerned, unfortunately there is no such body. In western and developed countries, the accreditation body recognizes the program of LIS and different organizations give 
preference to the candidates having passed professional education approved by accredited council.

The Higher Education Commission has developed LIS curriculum and, from time to time, revises it but the library schools, except one or two, develop their own curricula as per local requirements. The curricula of all Pakistani library schools are same with minor differences. All the universities are offering core courses to the students of BLIS or equivalent while specialized and elective courses are taught in next year. Although due to the demand, some of the courses have been bifurcated into theory and practical but there is no proper ratio of credits and marks between the two, nor do they have separate passing heads. The curriculum designed by HEC, indicates ratio between theory and practice but either the universities are not practicing the HEC curriculum or if practicing, they do not follow the standard ratio of HEC. 
Jhss, Vol. 9, No. 1 , January to June, 2018

\section{References}

Ameen, Kanwal. Challenges of preparing LIS professionals for leadership roles in Pakistan. Journal of Education for Library and Information Science. 47(3), 2006. pp.200-217.

Anwar, Mumtaz Ali. Asa Don Dickinson: The Founding Father of Modern Librarianship in British India. Pakistan Library Bulletin. 21 (2), 1990. p. 13.

Haider, S. J. Teaching of Cataloging and Classification in Pakistan. Cataloging \& Classification Quarterly, 43, 2006. pp. 53-65.

Khan, M. Tufail and Mahmood, Khalid (2013). A comparison of MLIS curricula taught in Pakistani library and information science schools with Higher Education Commission curriculum. Library Philosophy and Practice. 2013. Retrieved on 12 August 2007 from: http://works.bepress.com/khalid mahmood/18/

Khurshid, Anis. Library Education in South Asia. 20 (1-2). 1970. pp. 59-60.

Mahmood, Khalid. LIS Curriculum Review Using Focus Group Interviews of Employers. Library Philosophy and Practice, 2012.

Retrived on 12 August 2017 from http://digitalcommons.unl.edu/libphilprac/756/

Nagar,Murari Lal. Foundation of Library Movement in India. Ludhiana: Indian Library Institute and Bibliographical Center. 1983. pp. 2-3.

Sabzwari, G.A. Library education and manpower needs in Pakistan. Pakistan library bulletin. IV (3-4), 1972. pp. 55-71.

Warraich, Nosheen Fatima. MLIS Curriculum at Punjab University: Perception and Reflections. Library Philosophy and Practice, (e-Journal), March 2010.

Yusuf, M. Critical view of the courses offered at library schools in Pakistan. PLA journal. 18, 1996. pp. 68-76. 